\title{
Des graines, des fibres et des bobines
}

Mémoire d'une filière textile artisanale dans le Dendi (Nord Bénin)

Seeds, fibres and bobbins. Memoirs of a textile craft in Dendi, North Benin

\section{Lucie Smolderen}

\section{CpenEdition}

\section{Journals}

Édition électronique

URL : http://journals.openedition.org/ethnoecologie/4356

DOI : $10.4000 /$ ethnoecologie.4356

ISSN : 2267-2419

Éditeur

Laboratoire Eco-anthropologie et Ethnobiologie

Référence électronique

Lucie Smolderen, «Des graines, des fibres et des bobines », Revue d'ethnoécologie [En ligne], 15 | 2019, mis en ligne le 30 juin 2019, consulté le 29 octobre 2019. URL : http://journals.openedition.org/ ethnoecologie/4356 ; DOI : 10.4000/ethnoecologie.4356

Ce document a été généré automatiquement le 29 octobre 2019.

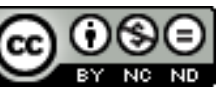

Revue d'ethnoécologie est mis à disposition selon les termes de la licence Creative Commons Attribution - Pas d'Utilisation Commerciale - Pas de Modification 4.0 International. 


\title{
Des graines, des fibres et des bobines
}

\author{
Mémoire d'une filière textile artisanale dans le Dendi (Nord Bénin) \\ Seeds, fibres and bobbins. Memoirs of a textile craft in Dendi, North Benin
}

\section{Lucie Smolderen}

\section{Introduction}

1 Le Dendi béninois se situe sur la rive droite du fleuve Niger (Figure 1), dans la région la plus septentrionale de l'actuelle République du Bénin. Caractéristique des savanes arbustives, le paysage est de nos jours principalement modelé par l'agriculture. Face à la pression démographique, la brousse recule sans cesse et les champs s'étalent à perte de vue d'un village à l'autre. Qu'il s'agisse des billons de la charrue ou des damiers de la culture irriguée, il est rare de trouver une parcelle de terre qui n'est pas travaillée. Hormis une petite section - entre Monsey et Kompa - de terres impropres à la culture, les sols alluviaux de la commune de Malanville et les sols ferrugineux lessivés de Karimama sont riches en matières organiques ${ }^{1}$. Le réseau hydrographique est constitué du fleuve, de ses trois affluents (d'ouest en est: le Kompa, l'Alibori et la Sota) et d'un tissu dense de cours d'eau temporaires, induisant la présence continue de l'eau dans la région, qu'elle soit en surface ou enfouie dans des nappes phréatiques.

2 Le climat de type soudano-sahélien présente deux saisons : l'une sèche (d'octobre à mai), l'autre pluvieuse (de fin mai à septembre). Le fleuve connait quant à lui deux crues : la première, appelée « eau blanche » (harri kware), correspond à la saison locale des pluies; la seconde, appelée « eau noire » (harri bi), est consécutive à la montée des eaux dans le delta intérieur du Niger au Mali (Van Driel 2001 : 52, 82).

Les habitants de la vallée ne s'y sont pas trompés, la fertilité du sol et l'accessibilité à l'eau font de cette région une terre de choix pour l'agriculture. Tout au long de l'année, les cultures commerciales, maraîchères et vivrières occupent les habitants de cette 
partie de la vallée. Parmi celles-ci, la culture du coton représente une part importante des revenus des ménages de la région du Nord (De Haan 1997).

Mise en place par l'administration coloniale française dans les années 1920 puis développée par l'état béninois indépendant à partir des années 1960, la filière cotonnière est devenue la figure de proue du développement. Avec la culture du coton devait suivre «le progrès », les infrastructures, la croissance économique. À l'échelle du pays, la filière cotonnière représente aujourd'hui $13 \%$ du PIB d'après PROCOTON ${ }^{2}$ (Wennink et al. 2013). Au vu de son importance économique mais aussi de ses implications sociales et politiques, de nombreux travaux d'économistes, d'agronomes ou encore de politologues se sont intéressés, de près ou de loin, à la filière coton béninoise (Tossou 1995, Ton 2004, Ale 2008, Kpadé 2011, Adjovo-Ahoyo et al. 2013, Wennink et al. 2013). Ces travaux de thèse de doctorat ou de recherche appliquée, commandités par des instances publiques ou privées, offrent un cadre structurel pertinent pour en comprendre les évolutions récentes (postindépendance), mais ne fournissent généralement qu'un aperçu très bref de sa trajectoire plus ancienne. Pourtant, derrière ces champs de coton ultra compétitifs, aspergés d'intrants chimiques, se cache l'histoire complexe d'une filière plus ancienne.

Nos travaux sur la filière textile du Dendi ont commencé en 2012 dans le cadre du projet ERC Crossroads of Empires ${ }^{3}$ dirigé par Anne Haour (University of East Anglia). Notre équipe, dirigée par Olivier Gosselain (Université libre de Bruxelles), travaillait sur les volets ethnographiques et historiques. Lors de cette première mission, l'un de nos objectifs était d'inventorier les artisanats représentés dans la région afin de pouvoir réaliser, dans un second temps, une étude historique de technologie comparée. Certains des artisanats dont il était question étaient encore pratiqués (comme la forge), d'autres étaient en passe de disparaître (comme la poterie), tandis que d'autres encore avaient complètement disparu. C'était le cas de la filière textile, aujourd'hui totalement abandonnée dans la région.

Nos entretiens auprès d'anciens cultivateurs, fileuses, tisserands et teinturiers ont montré à quel point cette filière artisanale avait été importante, tant du point de vue économique que culturel et social, pour ses nombreux acteurs et plus largement pour les habitants de la région. Depuis la fin du $19^{\text {ème }}$ siècle et certainement avant cela, la production de tissus en coton occupait un large réseau de personnes, aux occupations et statuts divers: le coton était planté par chaque chef de famille; le filage était l'activité féminine par excellence, pratiquée par toutes les femmes qu'elles soient esclaves ou nobles; la teinture à l'indigo était le propre de deux groupes (Kumaté et Goulmanchéba) spécialisés dans cette technique; tandis que les tisserands appartenaient à la classe servile. Parallèlement à nos enquêtes (plus classiques) portant sur l'histoire du peuplement de la région et sur les traditions orales ${ }^{4}$, la filière textile est devenue une porte d'entrée à travers laquelle interroger le passé de la région. En parlant avec d'anciens artisans, avec des femmes, ou encore avec des descendants d'esclaves, nous avons la possibilité de raconter «une autre histoire» (Olivier de Sardan 1976: 23): une histoire du quotidien, sociale, culturelle et économique, racontée à partir des marges de la société peu représentées dans les narrations historiques officielles monopolisées par les hommes de statut noble.

7 Dans cet article, le coton est l'acteur principal. Nous resterons au plus près des graines, des fibres, du fil, pour tenter de reconstituer la trajectoire de cette filière artisanale aujourd'hui disparue. Face à l'absence quasi-totale de sources historiques classiques 
(écrites ou archéologiques), le cadre temporel de l'étude est réduit. Nous nous focaliserons sur la période allant de la fin du XIX ${ }^{e}$ siècle au milieu du Xx $x^{e}$ siècle, période à laquelle l'administration coloniale met en place un programme cotonnier d'envergure. L'article propose un focus sur les deux premières étapes de la filière ${ }^{5}-$ l'obtention des fibres et le filage - sur lesquelles repose l'ensemble de la filière textile.

\section{Comment reconstituer une filière artisanale disparue?}

8 L'essentiel de cette étude repose sur des enquêtes ethnographiques ${ }^{6}$ menées systématiquement par l'auteur dans soixante-cinq villages de la vallée béninoise du fleuve Niger entre 2012 et 2018 (Figures 1 et 2)7. Au total, nous avons réalisé huit missions et quinze mois de terrain. Plus de six-cents enquêtes ont été menées dans cent-sept villages (voir les zones d'études Figure 2). En novembre 2015, un travail de terrain a été réalisé en collaboration avec l'archéobotaniste Louis Champion, qui réalisait à cette époque sa thèse de doctorat ${ }^{8}$. À cette occasion, nous avons travaillé plus spécifiquement dans vingt-six villages et réalisé quarante-deux enquêtes auprès de quatre-vingt-une personnes. Notre objectif était le suivant : retracer la trajectoire du paysage agricole du Dendi au cours du siècle dernier'. Ce travail de recherche nous a permis de mieux comprendre les évolutions (plus ou moins) récentes des pratiques agricoles, parmi lesquelles la culture du coton occupait une place primordiale tant dans le temps que dans l'espace. En effet, comme nous le verrons ci-dessous, le coton et ses produits dérivés jouaient un rôle commercial important dans la région, même avant l'intervention française au milieu du $\mathrm{xx}^{\mathrm{e}}$ siècle.

9 Tout au long de nos enquêtes de terrain une attention particulière a été portée aux situations d'enquêtes et aux rencontres avec les anciens praticiens (Bonnot 2018). Nos entretiens agissent comme un dispositif au sein duquel les remémorations techniques se donnent à voir. Nos recherches antérieures ont montré que lorsqu'un artisan partage son savoir, il ne raconte pas seulement une suite d'actions, il revit son activité : il mime, reproduit le son, utilise des substituts ou encore d'anciens objets qu'il a conservés (Smolderen \& Minguet 2013, Gosselain \& Smolderen 2016). Pour le chercheur, cette situation de remémoration technique multimodale est particulièrement intéressante puisqu'elle lui donne accès aux modes d'attachement et à la trajectoire intime qui unissent un ancien praticien à ses objets et son activité. Par ailleurs, ces objets - outils de tisserand ou de fileuse, bobines de fil, bandelettes de coton, pagnes, etc. - surgissant spontanément lors de nos enquêtes, fournissent aussi leur lot d'informations techniques sur les différents processus techniques constituant la filière textile.

10 Le cadre spatial de l'étude est bien défini puisqu'il se limite à la région du Dendi. Toutefois des observations et des enquêtes ont également été menées dans quatre autres zones (dans les communes de Banikoara et de Kandi au Bénin et autour des communes de Gotheye et Falmey au Niger), régions dans lesquelles certains segments de la filière textile sont encore pratiqués aujourd'hui (Figure 1).

11 L'observation de ces techniques en action a permis d'alimenter nos discussions avec les anciens praticiens dendi et de mieux comprendre les spécificités de chacun des processus techniques concernés (notamment celles du filage, du tissage et de la teinture). 
Le cadre chronologique de l'étude est plus flou. Dans l'état actuel de nos connaissances, il est impossible de proposer une date d'introduction ou d'apparition de la filière textile artisanale dans le nord du Bénin. Des découvertes archéologiques laissent penser qu'une espèce de Gossypium sp. poussait dans la région il y a de cela plusieurs siècles (Champion \& Fuller 2018). Les fusaïoles retrouvées en fouilles durant le projet Crossroads of Empires suggèrent que l'activité de filage a existé dans la région (Magnavita 2018, Tchibozo 2018). Toutefois le nombre d'occurrences est très faible et l'ancrage chronologique incertain. Ces découvertes ne permettent pas de tirer des conclusions quant à la date d'introduction de cette technique dans la région (Smolderen 2018 : 77). Concrètement, les sources orales (issues de nos enquêtes) et les sources écrites (archivistiques, historiques ou scientifiques) dont nous disposons ne nous permettent pas de remonter au-delà des dernières décennies du XIX $x^{e}$ siècle. Grâce à la juxtaposition de ces diverses informations, il devient possible de reconstituer l'état de la filière cotonnière et textile "d'avant les blancs ", pour reprendre l'expression couramment utilisée par nos informateurs. Le cadre chronologique ne peut donc pas se donner de manière absolue. Au contraire, il reste relatif à l'intervention française dans la filière cotonnière du Dahomey (actuelle République du Bénin).

Figure 1 : Zones d'enquêtes : le Dendi est la zone d'étude principale, les communes de Banikoara, Kandi, Falmey et de Gotheye sont des zones secondaires d'enquêtes destinées aux comparaisons

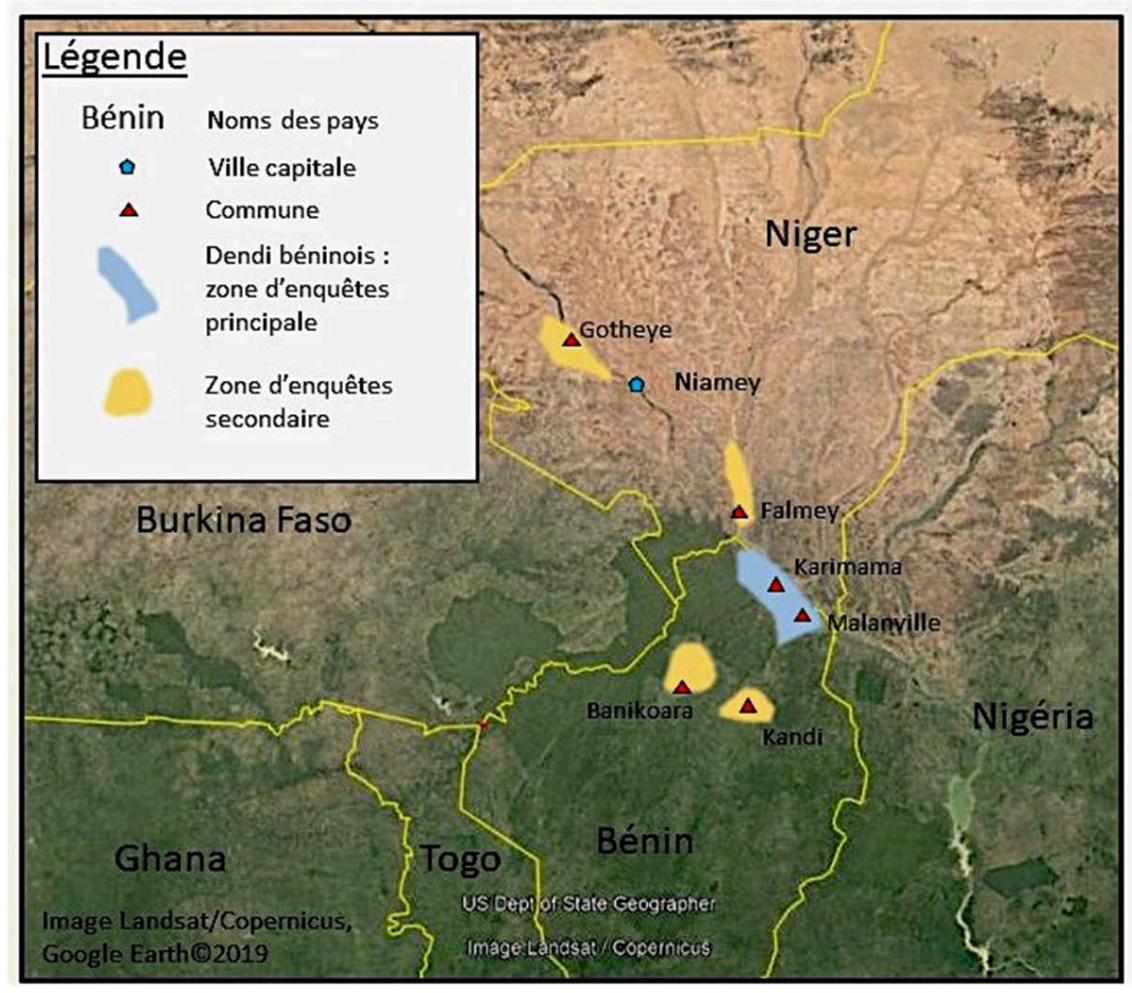

Carte L. Smolderen/Google Earth 
Figure 2 : Lieux principaux d'enquêtes (Dendi béninois) et anciens sites de teinturerie

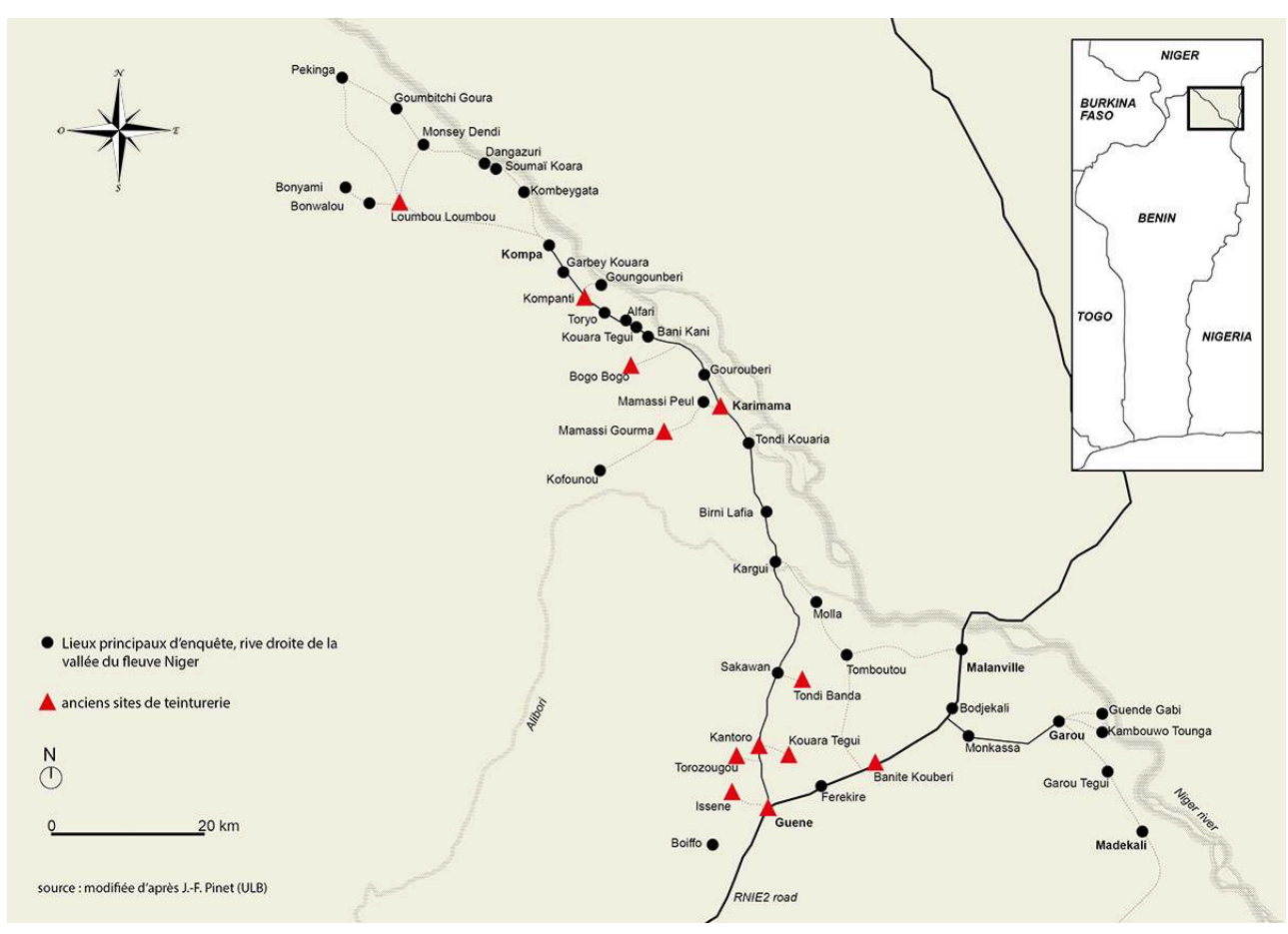

Carte L. Smolderen et J.-F. Pinet

\section{La production cotonnière précoloniale dans le Dendi}

\section{Contexte général et sources historiques}

Dès la deuxième moitié $\mathrm{du} \mathrm{XIX}^{\mathrm{e}}$ siècle, la question cotonnière occupe une place centrale dans les projets coloniaux français, anglais et allemand. Au début des années 1860, l'Europe doit faire face à " la disette du coton » qui émane du blocage des ports d'exportation américains (Duval 1864 : 5). Aux États-Unis, la guerre de sécession bat son plein. Or, l'Angleterre et surtout la France dépendent excessivement du coton des états confédérés (Sainlaude 2011: 15-16). Lorsque la guerre civile est déclarée, le président Lincoln instaure un embargo bloquant toutes exportations à partir des états confédérés (Roberts 1996: 67). Sans le coton américain, les puissances européennes ont plus que jamais besoin de sécuriser leurs importations en coton (Roberts $1996: 6$ ).

Privé de son «or blanc », la France entre dans une crise cotonnière sans précédent (Sainlaude $2011: 16-17)$. Finalement, c'est vers les colonies africaines que les regards et les espoirs français vont se tourner. D'après Céleste Duval, ancien inspecteur de la colonisation d'Algérie :

«Les contrées centrales, le Soudan, la Nubie, la Guinée, le Congo, le Dahomet...[sic], les îles africaines de Madagascar, dont les traités de 1815 nous garantissent une possession traditionnelle, et de Bourbon, sont des terres à coton. C'est dans ces contrées, c'est là que le cotonnier croît naturellement à l'état herbacé et en arbre ; c'est là qu'il se trouve en abondance, quoique dans certaines d'elles les indigènes n'en fassent aucun usage ; là, il croît partout, et les habitants connaissent sa culture depuis l'origine des temps! »(Duval $1864: 7$ ) 
Duval est optimiste. Grâce à ses colonies africaines, la France aurait les moyens de redresser sa propre économie, mais également de s'imposer comme le numéro un de l'exportation cotonnière en Europe, au point de faire de celle-ci « sa tributaire » (Duval $1864: 67)$.

16 À la fin du xixe siècle, les conflits internes qui animent depuis des décennies les différentes régions d'Afrique de l'Ouest se calment peu à peu (Roberts 1996 : 64-65). La «mission civilisatrice » de la France touche à sa fin et il est temps de penser à mettre en valeur les ressources des terres coloniales. Dans un rapport au ministre des colonies George Trouillot, daté du 24 septembre 1898, le colonel président Edgard de Trentinian s'exprime en ces termes :

«Jusqu'à ce jour, ni le temps, ni le plus souvent, les connaissances techniques, n'ont permis aux officiers et aux fonctionnaires du Soudan de faire des études approfondies, indispensables aussi bien à l'administration de la colonie qu'à sa mise en valeur. Au moment où le Soudan va jouir des bienfaits d'une paix définitive dans la plus grande partie de son territoire, il paraît opportun d'y faire effectuer des études aussi complètes que possible par des hommes techniques [...].» (Archives Nationales d'Outre-Mer (ANOM), FM-SG-SOUD.III.4)

Ce qui est entreprit pour le Soudan français (actuel Mali) l'est également pour le reste des territoires coloniaux. Des experts sont envoyés sur le terrain par l'administration coloniale. Ainsi, en 1894-1895, le commandant Toutée est placé à la tête d'un programme de recherche géographique, ethnographique et scientifique, qui sillonne les contrées du Moyen-Niger. Dans son rapport au ministre de l'Instruction Publique, il souligne, entre autres, les problèmes liés à l'acheminement du coton de l'intérieur des terres vers la côte. Il remarque aussi que le coton ne peut pousser trop près du littoral car le régime de pluie ne convient pas aux cotonniers qui, dit-il, "exigent, pour être récoltés dans de bonne condition, une sécheresse de cinq à six semaines [...]" (Toutée $1897: 139,1899$ : 27-28).

Parallèlement à ces programmes de recherches généraux, des missions focalisées sur la question cotonnière partent également pour le Sénégal, le Mali et la vallée du Niger. Au tournant du siècle, le contrecoup du choc cotonnier américain des années 1860 se fait toujours sentir et il devient impératif de trouver une solution à long terme. est emblématique de la situation coloniale française à cette époque. Après la course effrénée entre les puissances anglaise, allemande et française pour l'annexion des territoires, la compétition se situe maintenant à un autre niveau : celui des ressources. Le Docteur Yves Henry, agronome attaché au Jardin Colonial, part aux États-Unis en 1900 afin de trouver des semences appropriées aux sols français d'Afrique. Ces études visent certes à mettre en place une exploitation du coton indépendante du fournisseur américain, mais depuis peu, l'enjeu est aussi celui de la concurrence entre les puissances coloniales allemande et anglaise (Notes de l'inspecteur général de l'agriculture coloniale, du 13.06.1901 et du 09.10.1901, ANOM, FM-SG-SOUD.III.4).

La petite expédition, composée de trois membres, se rend ainsi au Mali, Bénin (ancien Dahomey) et en Sénégambie ${ }^{10}$ pour dresser des cartes de répartition des zones cotonnières autochtones et établir un programme de cultures expérimentales à partir des semences importées d'Amérique (Henry 1906).

21 À ce titre, le Dahomey offre des possibilités intéressantes : le territoire présente en effet plusieurs zones cotonnières où le coton est déjà cultivé afin d'alimenter l'artisanat 
textile local. D'après les observations d'Henry, la région la plus propice à la culture du coton est celle du " Moyen-Dahomey " (Henry 1906 : 198-199).

Dans le Haut-Dahomey ${ }^{12}$ le coton est aussi cultivé. Le Lieutenant de vaisseau Bretonnet (Martinière 1898: 91) et le Capitaine de génie Cambier (Trouillet 1905) avaient tous deux relevé la présence du coton dans ces contrées septentrionales à la fin du $\mathrm{XIX}^{\mathrm{e}}$ siècle. Le premier s'était contenté de souligner l'existence d'une culture cotonnière dans le nord de la colonie, tandis que le second avait fourni une description relativement détaillée de la pratique culturale au nord de Kandi. Voici ses propos rapportés dans un numéro de La Dépêche Coloniale Illustrée (1905 5(9) : 119-120) :

«[...] au nord de Kandi, et dans la vallée du Niger, il existe des champs de coton. Aussitôt que les premières pluies ont ameubli la terre, alors que l'igname élève déjà sa tige, les graines de coton ( 3 ou 4 par trou) choisies parmi les plus sèches et les plus fermes, sont semées presque à fleur de terre sur les versants des cônes, débarrassés [...] de toutes les herbes. Dans les champs, les graines sont semées sur deux rangées parallèles dans chaque sillon. La plante est buttée dès qu'elle atteint 15 ou 20 centimètres, puis elle est abandonnée à elle-même jusqu'à la récolte. L'indigène se borne à arracher les mauvaises herbes. L'écimage et l'émondage sont absolument inconnus. La récolte se fait lorsqu'elle est abondante et lorsque tout est mûr. Les gousses séchées au soleil pendant cinq à six jours sont ensuite égrenées à la main par les femmes et les enfants. Cette manière de procéder occasionne beaucoup de perte. »

La référence aux champs d'ignames (Dioscorea sp.) nous conduit à penser que ce mode cultural prévalait principalement dans la plaine de Kandi, où la culture d'igname était bien mieux représentée qu'elle ne l'était dans le Dendi, pays producteur de petit mil (Pennisetum glaucum), de sorgho (Sorghum bicolor) et de maïs (Zea mays). En effet, comme nous le verrons par la suite, dans la vallée du fleuve, les informateurs n'ont pas évoqué ce mode de culture intercalaire.

Malgré l'existence d'une culture du coton attestée dans le Haut-Dahomey, le Docteur Henry n'encourage pas la mise en place d'une exploitation coloniale dans la région. Le coton pousse dans les plaines du Borgou et de Kandi, mais d'après le Docteur Henry, la nature des sols et le manque d'intérêt des cultivateurs locaux pour ce type d'exploitation ne permettent pas d'envisager la culture coloniale du coton dans cette région (Henry 1906 : 197-206). Qui plus est, l'absence de voie de communication entre l'intérieur des terres et la région côtière ne facilite pas l'exploitation des ressources des contrées intérieures (Reste 1934).

Même si les (rares) sources historiques existantes ne nous permettent pas d'identifier avec précision les espèces de Gossipyum sp. cultivées dans le Dendi avant l'intervention coloniale, elles nous laissent imaginer que la culture du coton existait bien dans la vallée béninoise du fleuve Niger à la fin du XIX ${ }^{e}$ siècle. Sans pouvoir répondre à la question de l'identification de manière précise, plusieurs éléments issus cette fois de nos enquêtes peuvent nous permettre de reconstituer cette étape initiale de la filière et de proposer une hypothèse.

\section{Acquisition initiale de la matière première}

Pour l'ensemble de la filière, la matière première est toujours le coton : qu'il s'agisse de la graine pour la culture, des fibres pour le filage, des fils pour le tissage, des bandes d'étoffes de coton pour les pagnes... En ce sens, chacune des étapes du processus 
technique produit la matière première de l'étape suivante. Cependant, la filière toute entière repose bien sûr sur une étape d'acquisition initiale : celle de la production du coton. Celle-ci s'avère donc essentielle.

D'après nos interlocuteurs, deux espèces de coton ont été cultivées dans la région avant l'intervention coloniale, au milieu $\mathrm{du} \mathrm{xx}^{\mathrm{e}}$ siècle. Elles présentent chacune un mode cultural et des caractéristiques propres. Le cotonnier le plus ancien du Dendi était appelé habou sando ce qui signifie littéralement « coton dur » en dendi ${ }^{13}$. Cette dénomination fait référence à la difficulté qu'éprouvaient les fileuses à retirer les graines des fibres ${ }^{14}$. Ces dernières étaient également moins blanches que celles du coton actuel. La corolle, quant à elle, avait une couleur rouge ${ }^{15}$. Ce coton était cultivé de manière pérenne : après la récolte le pied était laissé en terre ; s'il survivait à la saison sèche, il pouvait ainsi donner plusieurs années de suite (entre deux et sept ans d'affilée selon les témoignages) ${ }^{16}$. Les plants âgés de deux ans et plus atteignaient alors une taille importante dépassant celle de l'homme ${ }^{17}$. Les plus grands donnaient même de l'ombre ${ }^{18}$. Ils devenaient alors comme des arbres dans lesquels les enfants grimpaient pour récolter les capsules les plus hautes ${ }^{19}$.

Au niveau du mode de culture, les informations sont variées. Habou sando pouvait être cultivé en champ, selon les besoins de la famille. Ce mode de culture pérenne en champ rappelle ce que Heinrich Barth observa lors de son voyage vers Kano (non loin de l'embranchement de Tessaoua au Niger) (Barth 1860 : 314) :

" Nous fîmes, ce même jour, deux nouvelles découvertes du règne végétal, en rencontrant les premiers champs de coton et le premier tulipier. [...]; les champs de coton, qui nous révélaient de nouveaux aperçus de l'industrie indigène, rompaient agréablement avec l'uniformité des champs de blé. Les arbrisseaux qui les composent sont presque toujours en frondescence tandis qu'il s'en trouve quelquesuns en fleurs et d'autres à l'état de maturité. Malheureusement les jeunes plantations seules étaient cultivées avec soin. »

Dans le champ de culture pérenne, certains des plants sont déjà âgés d'un, deux ou trois ans tandis que d'autres sont nouvellement plantés ${ }^{20}$. Cette méthode occasionne un décalage dans le développement des différents plants. Mais plutôt que de la négligence, comme le suggère Barth, il pourrait s'agir d'une manière de profiter au maximum de ce que chaque graine a à offrir, une stratégie mise en place pour répondre à la difficulté de s'approvisionner en graines (infra).

31 Les hommes de la famille travaillaient la terre à la houe (daba) puis formaient des lignes de poquets. Les femmes et les enfants y versaient une petite poignée de graines avant de les recouvrir légèrement de terre d'un coup de talon. Une fois les pousses sorties, elles étaient démariées pour ne laisser que deux plants par poquets ${ }^{21}$.

Parallèlement à la culture en champ, d'autres témoignages évoquent une culture d'ordre domestique. Quelques plants de habou sando étaient semés dans la cour des concessions, derrière les habitations ${ }^{22}$. Cette différence d'échelle peut s'expliquer par un accès à la terre et une main d'œuvre réduits au sein de la maisonnée concernée. Comme le souligne Champion dans sa thèse (Champion 2019: 414-419), le coton n'est pas une culture de subsistance alimentaire comme le sont les cultures vivrières, telles que le mil et le sorgho. Sa mise en place et son développement reposent sur un surplus de terre et de main d'œuvre. Cette différence d'échelle entre une culture en champ et une culture domestique pourrait également s'expliquer par le manque de sol propre à la culture cotonnière. 

siècle dans le Dendi, sont limitées. Ce cotonnier était cultivé annuellement ${ }^{28}$. Il était planté vers le mois d'août après la récolte du petit mil hâtif, dans le même champ. Il était prêt à être récolté trois mois plus tard. Sa corolle était jaune et ses fibres blanches ${ }^{29}$. Par opposition au grand cotonnier habou sando (décrit ci-dessus), ce coton se caractérisait par sa petite taille ${ }^{30}$. Les informateurs s'y référaient généralement en utilisant le terme générique habou (coton), ou en spécifiant parfois andasaraa habou (coton des blancs). Les caractéristiques de ce second cotonnier font penser qu'il s'agit d'une espèce de coton commerciale importée. En effet, comme nous le verrons cidessous, dans les années 1920-30, l'administration coloniale commence à s'intéresser de plus près au territoire septentrional de la colonie dahoméenne. Plusieurs essais de plantation de graines importées sont réalisés, sans pourtant qu'un suivi systématique soit assuré. Les cotonniers de petites tailles décrits ci-dessus seraient alors le résultat de ces premiers essais. Toutefois, il pourrait aussi s'agir d'une variété hybride. Les recherches agronomiques sur le coton ont très tôt montré le haut niveau d'hybridité du Gossypium sp. (Réteaud 1924 : 209, Reste 1934 : 136). Le commerce du coton avec des régions plus méridionales (infra) pourrait être à l'origine d'une telle hybridation.

\section{Organisation de la filière cotonnière artisanale}

Quel que soit le type de coton cultivé (pérenne, annuel, de petite taille ou arborescent), l'organisation de la production cotonnière était toujours la même et répondait à une division genrée du travail. Le travail agricole était géré par les hommes de la famille. Les femmes et les enfants étaient présents pour prêter main forte lors des semis et des récoltes, mais toutes les autres tâches agricoles étaient effectuées par les hommes (préparation $\mathrm{du}$ sol, binage, sarclage, etc.) ${ }^{31}$. La division genrée du travail n'était d'ailleurs pas limitée au domaine cotonnier mais régissait toute l'organisation du travail agricole quelle que soit la culture. Jusqu'à une époque récente (vers 1980), les femmes dendi ne cultivaient pas leurs propres champs et ne participaient que de manière ponctuelle et marginale aux travaux champêtres. Elles avaient en revanche à leur charge l'ensemble des activités domestiques parmi lesquelles la transformation des produits agricoles occupait une place importante (préparation des repas, filage des fibres de coton).

Malgré cette apparente division sexuelle, le coton avait un statut particulier parmi les nombreuses cultures anciennes du Dendi. À l'inverse des produits vivriers (sorgho, 
maïs, petit mil hâtif et tardif) dont le stockage et la distribution étaient entièrement gérés par les hommes, le coton, une fois récolté, était partagé entre les femmes du ménage. Elles le stockaient directement dans leur chambre dans de grands paniers en feuilles de rônier (appelés djogo) ${ }^{32}$, des marmites ou des calebasses ${ }^{33}$. Chacune des femmes de la maisonnée gérait sa réserve afin de répondre aux besoins de la famille, filait le coton et apportait le fil au tisserand pour réaliser le textile qui habillerait sa famille. Une fois sorti du champ, le coton devenait « une affaire de femme».

Avant de se mettre à l'ouvrage, la fileuse commençait toujours par l'étape préliminaire de l'égrenage (cf. "processus technique», pl. 1). Cette étape, cruciale pour tout le maintien de la filière, permettait la récupération des graines en vue de la saison prochaine ${ }^{34}$.

Plusieurs informateurs ont évoqué la difficulté de s'approvisionner en graines ${ }^{35}$. Lors de l'égrenage celles-ci pouvaient casser. Par ailleurs, les graines n'étaient pas toutes de bonne qualité $e^{36}$. La culture pérenne permettait également de minimiser l'impact de cet approvisionnement difficile en profitant au maximum de chaque graine. La qualité de la récolte était enfin fonction de la qualité du sol et de la pluviométrie ${ }^{37}$ (Reste 1934 : 131,136). Lorsque la récolte n'était pas suffisante pour couvrir tous les besoins en coton de la famille, il fallait parfois recourir à une autre filière d'approvisionnement.

\section{Le coton, une marchandise régionale}

41 À côté de la culture locale, l'approvisionnement en coton pouvait également se faire auprès de commerçants baribas, venus de la région de Banikoara, qui vendaient du coton dans les marchés dendi ${ }^{38}$.

Chargés de grands paniers remplis de coton, ces colporteurs remontaient la piste reliant la région de Banikoara au Dendi (à travers l'actuel parc du W) et vendaient leur coton dans les villages du plateau et de la vallée du fleuve. Étant donné la facilité avec laquelle le coton peut s'hybrider, ce commerce de coton vendu non-égrené pourrait être à l'origine de l'hybridité de certaines variétés locales.

Lorsqu'une récolte avait été particulièrement fructueuse, les cultivateurs dendi vendaient également leur surplus sur les marchés ${ }^{39}$. Lors de ces transactions, les principaux clients étaient les femmes qui complétaient de cette manière leurs réserves de coton à filer ${ }^{40}$. Les femmes peules, dont les époux essentiellement tournés vers l'élevage ne produisaient pas de coton, se procuraient ainsi du coton sur les marchés ${ }^{41}$. Les peuls ${ }^{42}$ réalisaient également beaucoup d'échanges avec les autres groupes ethnolinguistiques de la région ${ }^{43}$, donnant du petit bétail contre des textiles (fils, bandes, et pagnes).

De nombreux commerçants dendi et nigériens, notamment zarma, achetaient du coton en gros dans les marchés de la rive droite du fleuve, pour le revendre de l'autre côté du fleuve, au Niger, dans des régions plus inhospitalières à sa culture ${ }^{44}$. Situé au carrefour entre plusieurs écosystèmes différents, le Dendi était une plateforme commerciale de choix. Cette caractéristique ne manqua pas d'attirer les commerçants, notamment hausa, qui longeaient le fleuve avant de continuer leur route vers Toumbouctou ou vers le Burkina Faso ${ }^{45}$ (infra). 


\section{Fil de trame et fil de chaîne : le maillage du social}

\section{Le processus technique du filage}

Le filage a aujourd'hui disparu dans la région étudiée. Nous proposons ici une reconstitution en image du processus technique basée sur nos enquêtes orales et sur les mises en scène auxquelles les anciennes fileuses se sont elles-mêmes prêtées lors des enquêtes (Smolderen \& Minguet 2013). Tout système technique étant lui-même intégré à un univers social plus large, la reconstitution des grandes étapes techniques du filage est un préambule nécessaire pour comprendre l'ancrage social du système technique développé ci-dessous (Lemmonier 2010).

Deux planches sont consacrées à cette reconstitution: la première détaille les trois étapes de la phase préparatoire ainsi que l'étape alternative du cardage (Figure 3); la seconde détaille la phase de façonnage, l'étape alternative du façonnage du fil de chaîne, et la phase du renvidage (Figure 4).

Figure 3 : Phase préparatoire : étape du nettoyage, étape de l'égrenage, étape du battage, étape alternative du cardage

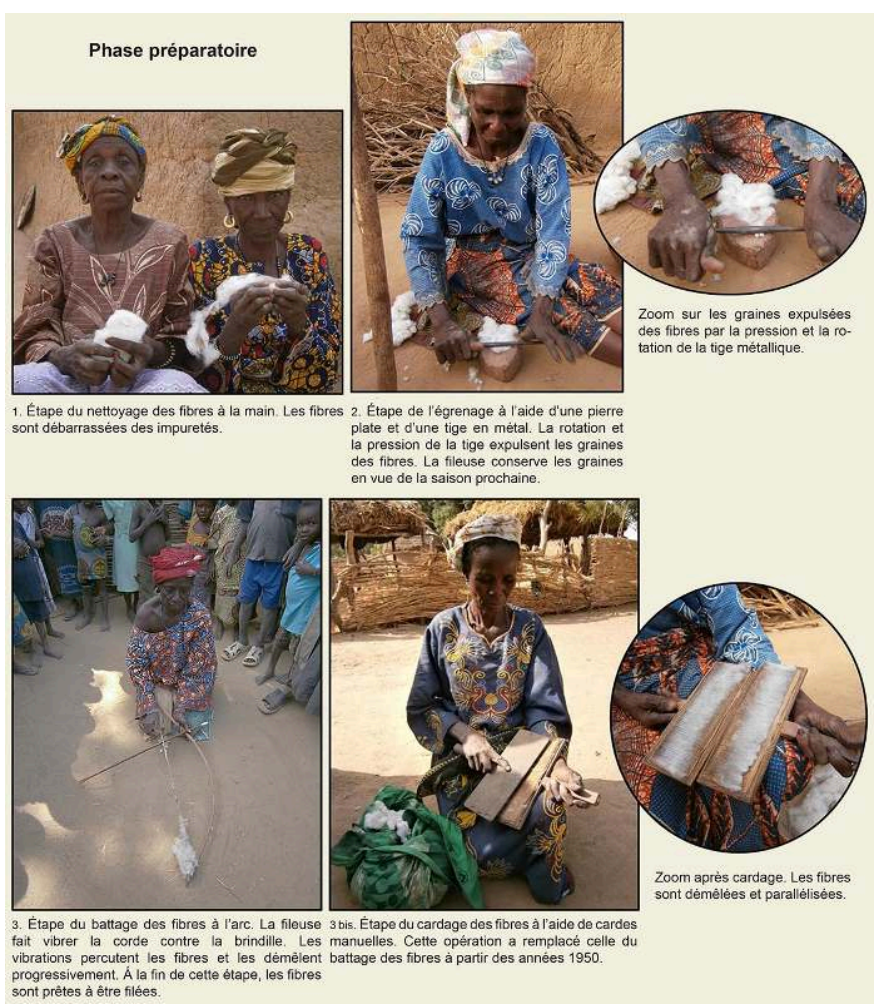

(c) L. Smolderen 
Figure 4 : Phase de façonnage : étape de la connexion fibres/fuseau, étape de la torsion, étape alternative du façonnage de fil de chaîne. Phase de renvidage : étape du renvidage

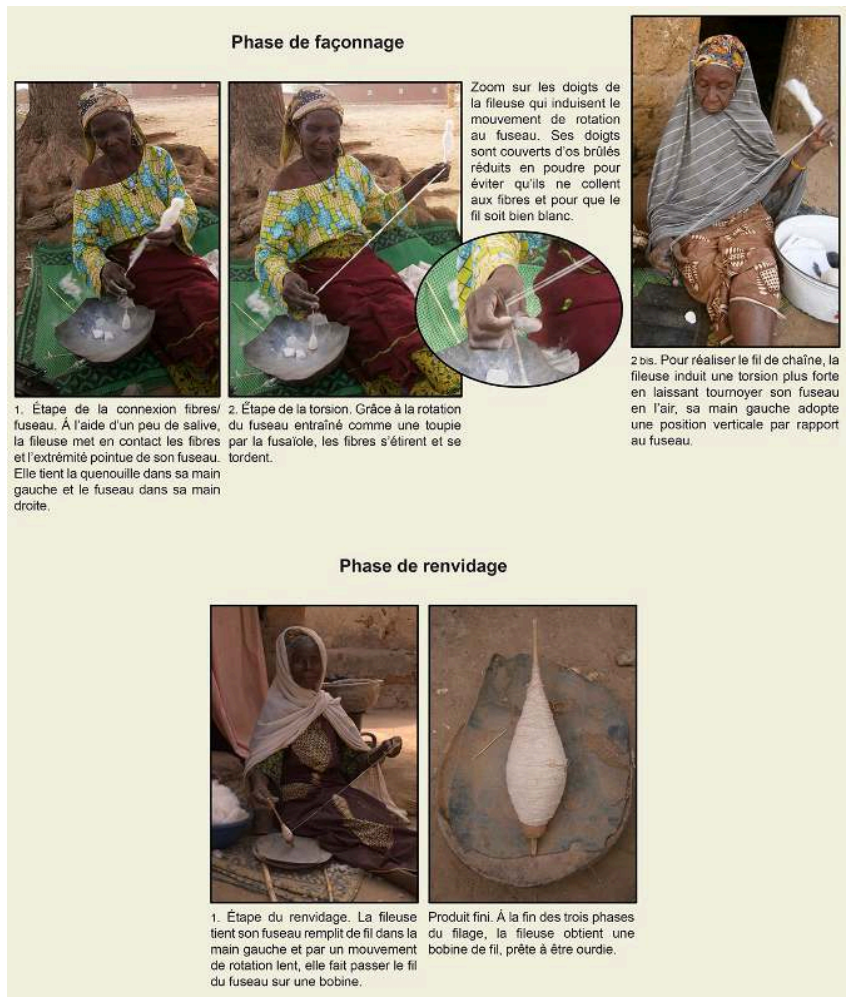

\section{(c) L. Smolderen}

Lors de ces mises en scènes nous avons eu l'occasion de voir en action le nécessaire à filer. Ce dernier se compose d'une série d'objets que nous présentons brièvement ici. Le dispositif à égrener est composé de deux parties (Figures 3 et 4). La pierre plate (tondi), partie passive de ce dispositif, a une surface lisse parfois rendue légèrement concave par usure. Elles se trouvaient dans la brousse ou près du fleuve. La tige en métal (guuru), quant à elle, s'achetait chez le forgeron. Cette tige est légèrement renflée en son centre tandis que les extrémités sont plus minces. Cette forme particulière facilitait la rotation de la tige sur les fibres.

La fileuse utilisait ensuite le bindigi, un arc à battre le coton (Figure 3-3). Celui-ci est fabriqué par ses propres soins à l'aide d'une tige de bois souple et d'un fil de coton enduit de poudre d'os brûlé (karou). Le fil de coton est bandé entre les deux extrémités du bois souple de manière à former un arc. La fileuse utilise ensuite une petite baguette de bois pour faire vibrer la corde de l'arc. Dans les années 1950, l'arc à battre le coton a été remplacé par les cardes manuelles ( $k a d a)$. Celles-ci sont composées de deux brosses à dos plat en bois avec des dents métalliques (Figure 3-3bis). Toutes les femmes ne possédaient pas leur propre arc à battre le coton ou pierre à égrener et devaient alors les emprunter à une voisine ou une amie. En revanche, chacune devait impérativement posséder son propre fuseau (handjel), pièce maîtresse du nécessaire à filer. Le fuseau se compose d'une brindille fine aux extrémités pointues, mais aux bouts arrondis. Dans la partie inférieure du fuseau, une boule d'argile cuite de forme globulaire ou légèrement ovalaire sert de volant d'inertie, appelé fusaïole (Figure 4 " phase de façonnage »). Ces fuseaux pouvaient s'acheter auprès des potières. 
Lors de l'opération de tordage des fibres, la fileuse enduisait ses doigts d'une poudre d'os brûlé et pilé fabriquée par ses soins ou par une des femmes âgées de la concession. Cette poudre, utilisée comme un talc, évitait que les doigts ne collent aux fibres, à cause de la transpiration. La poudre d'os permettait aussi d'obtenir un fil bien blanc. Une fois l'opération de tordage terminée, la femme renvidait le fil de son fuseau sur une bobine (handjel beri). Celle-ci était fabriquée à l'aide d'une brindille épaisse et d'un bouchon d'argile séchée, qui sert à la fois à la rotation de la bobine mais aussi à empêcher que le fil ne se dévide (Figure 4-2, « produit fini »).

\section{Le travail des fibres au cœur de l'identité féminine}

50 Dans le Dendi comme dans les régions adjacentes, le filage était une activité féminine (Étienne-Nugue 1984: 180, Olivier de Sardan 1984: 55. Voir aussi Boussari Vokouma (1999) et Gbinsa (1991)). À l'inverse des activités de poterie, de forge ou de tissage qui définissent un groupe socio-professionnel servile (Olivier de Sardan 1984), le filage était réalisé par toutes les femmes sans distinction de statut ou de classe. Il s'agissait par ailleurs d'une activité artisanale hautement spécialisée qui nécessitait une longue période d'apprentissage (Kriger $2006: 10,90-91$ ).

51 Filer se faisait parfois durant la journée, lorsque les autres tâches ménagères étaient terminées, mais les femmes filaient surtout en groupe pendant la soirée, voire même jusqu'au petit matin ${ }^{46}$. La nuit contribuait également à créer une ambiance particulière. Toutes les femmes d'une concession ${ }^{47}$, fillettes et grands-mères, se retrouvaient pour filer ensemble autour du feu. Des voisines se joignaient au groupe. Tout en travaillant, les femmes chantaient et causaient ${ }^{48}$.

52 Lors de son premier voyage, Mungo Park raconte un épisode au cours duquel il s'est reposé dans la case d'une femme qui l'a recueilli alors qu'il était affamé (Lanoye 1860 : 56) :

«[...] elle m'invita à prendre du repos sur une belle et fraîche natte qu'elle étala dans un coin de sa hutte pendant qu'à l'autre extrémité elle réunit autour de sa lampe un certain nombre de jeunes négresses, ses exclaves ou ses compagnes, et elle se mit à filer du coton avec elles. Leur veillée se prolongea fort avant dans la nuit, et, comme toutes les fileuses du monde, elles mêlèrent à leur travail des récits et des chants. Ces derniers cadencés légèrement et à demi-voix [...] ».

53 Certes, Mungo Park se trouvait à ce moment-là dans la région de Ségou au Mali, mais l'ambiance qu'il décrit correspond à l'idée que l'on peut se faire des veillées de fileuses du Dendi. "En filant on causait, moi-même j'ai appris quelques histoires à ces occasions-là, en m'asseyant à côté des vieilles. $»^{49}$ se rappelle l'une de nos interlocutrice. La lenteur de l'activité et les gestes répétés laissent la place au discours, tandis que l'ambiance intimiste du feu est propice aux narrations, aux échanges intergénérationnels et aux confidences entre paires.

54 Le filage, dont la pratique était individuelle - puisque le processus technique était exécuté par une seule personne - mais collective - puisqu'elle se pratiquait en groupe n'était donc pas seulement un acte technique : il s'agissait également d'un acte social. Filer ne se résumait pas à fabriquer du fil. Par la pratique, les jeunes fileuses s'inséraient dans la société.

Dès l'âge de quatre ou cinq ans, les fillettes s'asseyaient auprès des autres femmes de leur concession et les observaient travailler durant de longues heures. Par l'observation 
d'abord puis par la pratique, elles apprenaient progressivement les différentes phases du processus selon un schéma classique d'apprentissage périphérique (Lave \& Wenger 1991, Smolderen 2018). La jeune apprentie commençait par le nettoyage des fibres, venait ensuite l'étape de l'égrenage et du battage des fibres ${ }^{50}$ (pl. 1). Ces étapes préparatoires demandaient peu d'aptitudes techniques et représentaient donc une bonne entrée en matière pour les fileuses débutantes. Cette phase préliminaire relativement simple n'était pas pour autant moins importante que les suivantes - bien au contraire - puisqu'elle affectait elle aussi la qualité du produit fini (Kriger 2006:9). En outre, l'obtention d'un fil de bonne facture dépendait autant de l'aptitude technique de la fileuse que de la qualité de la matière première utilisée (longueur des soies, résistance et couleur des fibres) et de son habileté à l'évaluer. En nettoyant le coton de ses impuretés, l'apprentie entrait en contact direct avec la matière. Elle apprenait à distinguer les fibres de bonne ou de moins bonne qualité. Progressivement, la jeune fileuse devenait experte en la matière. Son expertise relevait alors autant du domaine technique que du domaine sensible.

Par la suite, l'apprentissage concernait l'étape centrale de la torsion des fibres (Figure 4). Dans un premier temps, la jeune fileuse - encore peu habile - réalisait un fil lâche et irrégulier. Celui-ci servait alors de fil de trame (sofou en dendi) ${ }^{51}$. Le fil de chaîne (djersé en dendi) devait quant à lui être particulièrement résistant et régulier. La torsion nécessaire à son obtention était bien plus importante que celle du fil de trame. Ce dernier était réalisé par les fileuses les plus expérimentées. Cette spécificité technique liée à la réalisation du fil de chaîne résonne d'ailleurs encore aujourd'hui. Lors de nos interviews, certaines de nos interlocutrices se sont présentées comme des fileuses capables de réaliser du fil de chaîne. Certaines accompagnaient ce témoignage d'un son qui imite le bruit du frottement des fibres sur le bois du fuseau. Ce petit bruit (frrrt frrrt frrrt) en est venu à symboliser la force de torsion et l'habileté de la fileuse ${ }^{52}$.

Ces moments de pratique collective ont marqué les mémoires et les corps des fileuses. À ce titre les témoignages de nos interlocutrices sont éloquents : "nous bavardions et nous chantions autour du feu, mais le filage n'était pas une partie de plaisir. C'était un travail difficile et douloureux. $\|^{53}$; « à force de filer, le fuseau déformait nos doigts. $»^{54}$; «j'ai filé jusqu'à n'avoir plus de peau sur les doigts. " $^{55}$; on n'avait pas le droit de bouger avant d'avoir fini un fuseau, sinon on se faisait taper fort. $»^{56}$. La pression exercée par les aînées sur les plus jeunes était intense. Le filage était une activité lente, au rendement bas. Par les menaces, les aînées s'assuraient de l'efficacité des plus jeunes. Sous le regard sévère des grands-mères, les jeunes filles apprenaient non seulement à filer mais également à travailler vite et bien. Elles incorporaient les valeurs d'obéissance et de travail essentielles à la figure de "la bonne épouse ». La pratique communautaire de l'activité n'agissait donc pas seulement sur la technique elle-même mais elle participait également au façonnage d'une identité féminine commune ${ }^{57}$.

« Les seuls jours de l'année où l'on ne filait pas, c'était quand il y avait des funérailles. C'est pourquoi tous les matins, on allait demander aux vieux si quelqu'un n'était pas mort durant la nuit. ${ }^{58}$. Le filage était au centre des préoccupations quotidiennes des femmes et des jeunes filles. "Le filage, c'était cela notre travail. Nous n'avions pas d'autres occupations que piler le mil et faire du fil $»^{59}$. Au même titre que le pilage du mil, le filage était considéré comme une activité de subsistance essentielle au maintien et à la reproductibilité du ménage. Il était donc fondamental pour une jeune fille de savoir filer avant de pouvoir se marier. 
59 Le jour du mariage, le nécessaire à filer (le fuseau, la poudre d'os brûlé et pilé, l'arc à battre le coton, la pierre à égrener (Figures 3 et 4)) faisait partie du trousseau que la jeune mariée amenait avec elle ${ }^{60}$. Durant la première moitié du $\mathrm{xx}^{\mathrm{e}}$ siècle, le trousseau était composé d'objets essentiellement utilitaires : un pilon et un mortier, des nattes, des marmites en terre cuite, un ensemble de calebasses pour travailler et manger, une jarre à eau et un nécessaire à filer. Une fois installée dans son nouveau ménage, la jeune épouse avait pour responsabilité de piler le mil pour nourrir sa famille mais aussi de filer suffisamment de bobines de fil pour l'habiller.

60 Le filage et sa culture matérielle, en participant à la construction d'une identité féminine socialement acceptée, assuraient la place de la femme dans son ménage et dans la société.

\section{Filage et tissage, ou la fabrique du lien social}

61 Le fil servait en premier lieu à vêtir les membres de la famille. D’après G. François, ancien chef du cabinet du gouverneur du Dahomey, la tenue portée par les dendi était constituée «de boubous haoussa plus ou moins ouvragés de broderies suivant la richesse et l'ostentation du propriétaire, d'un pantalon large resserré à la cheville, de sandales et du turban. » (François 1906 : 108). Néanmoins, les personnes décrites ici, en contact avec les colons, appartenaient le plus souvent à l'élite de la société. Leur mode vestimentaire ne correspondait donc pas forcément à celle suivie par les habitants de classes plus modestes. À la suite de la description fournie par G. François, on peut toutefois penser qu'au début $\mathrm{du} \mathrm{xx}^{\mathrm{e}}$ siècle les dignitaires et chefs de villages dendi s'étaient alignés sur les traditions vestimentaires du Nigéria. Cette mode, importée par les marchands hausa des centres textiles du Caliphat de Sokoto, était fortement associée aux coutumes islamiques ${ }^{61}$. À la fin du XIX ${ }^{e}$ siècle et durant les premières décennies $\mathrm{du} \mathrm{xx}^{\mathrm{e}}$ siècle, posséder et se vêtir d'un pagne importé du Sokoto était considéré comme un signe ostentatoire de supériorité sociale. Non seulement le pagne teint à l'indigo typique de la production du Sokoto ${ }^{62}$ faisait directement référence à une élite islamisée, mais il symbolisait également la capacité de son propriétaire à s'intégrer dans une économie de marché basée sur un échange monétarisé, ce qui n'était pas le cas des pagnes de fabrication locale qui s'échangeaient contre biens et services (infra).

62 Nos données offrent un autre éclairage sur les modes vestimentaires dendi. L'habillement communément porté par les gens de la région était constitué d'un pagne préférentiellement blanc pour les hommes, drapé en diagonale sur une épaule, et noir (bleu indigo) pour les femmes, qui le portaient noué autour de la poitrine ou de la taille $^{63}$. Ces pagnes étaient composés de bandes étroites (environ $10 \mathrm{~cm}$ de large) cousues parallèlement les unes aux autres, bord à bord. Les pagnes des hommes comptaient quinze bandes et ceux des femmes douze ${ }^{64}$. Les pagnes pouvaient être unis blanc ou noir - mais il existait aussi d'autres styles plus colorés faits de bandes de couleurs différentes obtenues grâce à des teintures naturelles, de bandes unies traversées longitudinalement par une ou plusieurs lignes de couleur (comme le pagne téra composé de bandes blanches traversées longitudinalement par des lignes rouges et noires), ou encore d'un enchevêtrement de fils noirs et blanc dans la trame (appelé tchengéré).

Malgré la simplicité stylistique apparente des pagnes tissés dans le Dendi jusqu'au milieu du $x^{e}$ siècle (Smolderen 2018), l'habillement était investi symboliquement par 
leurs propriétaires. Le nombre de bandes, la couleur, la qualité du tissu étaient autant de critères d'évaluation du textile, qui permettaient aux propriétaires de se situer socialement ${ }^{65}$. Par exemple : une jeune fille en âge de se marier portait un pagne de neuf bandes; une jeune fille trop jeune pour se marier portait un pagne de sept bandes; une jeune femme mariée qui n'avait pas encore eu d'enfant portait un pagne blanc; un homme qui voulait montrer sa richesse et la puissance de sa belle-famille portait son pagne de mariage ${ }^{66}$ - orné de plusieurs couleurs - sur la place publique ${ }^{67}$. En ce sens le textile était l'un des signes ostentatoires du statut social. La femme - en tant que fileuse - participait activement à la reproduction et à la visibilité sociale du ménage.

Le filage était une activité particulièrement fastidieuse. Afin de faire face à la lenteur de l'activité, les femmes s'organisaient en groupes d'âge et d'affinité pour former des tontines ${ }^{68}$ au sein de leurs concessions ou de leurs quartiers ${ }^{69}$. Le modèle classique de la tontine de fil était le suivant : tour à tour et selon un ordre prédéfini, chaque membre de la tontine apportait une grande calebasse de coton; le coton était divisé en parts égales entre chacune des femmes du groupe; elles filaient le coton qui leur avait été remis et une fois l'ouvrage effectué, elles remettaient l'ensemble des bobines à celle qui avait apporté le coton. De cette manière en un temps réduit, chaque membre de la tontine recevait tour à tour une importante quantité de fil. Ce mode d'organisation visait à augmenter la force de travail et à répondre stratégiquement à la lenteur de l'activité. Mais la tontine de fil, comme toutes les tontines, était aussi une manière d'entretenir et de matérialiser un réseau de solidarité qui dépassait l'activité en question, pour toucher à toutes les sphères de la vie quotidienne. En ce sens, si une femme ne respectait pas les règles de rétribution du service rendu, cette dernière risquait non seulement de se faire exclure de la tontine mais aussi de devenir un paria et d'être exclue de toute vie sociale au sein de son quartier.

Tout au long de l'année, les femmes géraient leurs réserves de coton et leur ouvrage en vue d'avoir suffisamment de fil lorsque les tisserands commenceraient leur activité. Le tissage était une activité saisonnière menée durant la saison sèche. Pendant la saison des pluies, les tisserands étaient agriculteurs. Il fallait donc attendre la fin des travaux champêtres et le début de la saison sèche, aux alentours du mois de janvier, pour que les tisserands du village et les saisonniers installent leurs ateliers. Les tisserands travaillaient sur des métiers horizontaux à pédales de type "soudanais ", caractérisés par l'étroitesse des bandes réalisées (Lamb 1975 : 75 ; Etienne-Nugue 1984 : 182). Les fileuses devaient apporter au tisserand les quantités de fils de trame et de fils de chaîne nécessaires à la réalisation de leur commande ${ }^{70}$.

Une fois le nombre de bobines suffisant, les fileuses devaient ourdir le fil. L'ourdissage était une étape longue et fastidieuse qui visait à la préparation de l'écheveau de fils de chaîne (Étienne-Nugue 1984: 155). L'écheveau devait présenter deux groupes de fils séparés pour former les nappes supérieures et inférieures de la chaîne. Une fois encore, cette étape de la filière reposait sur un réseau de solidarité sous-jacent à toute la vie sociale du quartier. Les fileuses faisaient appel aux autres femmes de leur concession ainsi qu'à leurs voisines pour leur venir en aide ${ }^{71}$. Pour former les deux nappes de fils de chaîne, il fallait dévider deux bobines de fil en parallèle, tout en formant un huit autour de piquets plantés dans la cour. L'espacement entre les piquets et, donc, la distance parcourue par le fil devait correspondre au nombre de bandes désiré (Gbinsa 1991 : 55, Boussari Vokouma 1999 : 110). L'opération de dévidage devait être reconduite 
plusieurs fois, jusqu'à l'obtention du nombre de fils de chaîne nécessaire pour la réalisation de la bande ${ }^{72}$. L'écheveau de fil était ensuite noué à l'endroit du croisement des fils, au centre du huit, afin que ceux-ci ne s'emmêlent pas et que les deux nappes, supérieure et inférieure, restent bien séparées. Cette étape pouvait durer plusieurs heures.

Une fois le fil ourdi, la fileuse apportait ses écheveaux chez le tisserand. Elle négociait alors le prix de la commande, le style, le nombre de bandes ${ }^{73}$. D'après certains de nos interlocuteurs, les hommes se rendaient parfois eux-mêmes chez le tisserand pour passer commande ${ }^{74}$. Cependant, même dans ce cas-là, il s'agissait toujours du fil et de la commande fournis par leurs épouses. Généralement, l'arrangement avec le tisserand consistait en un échange de biens et de services. Le prix des pagnes n'était pas fixe. Le payement consistait en un surplus de fil, grâce auquel le tisserand fabriquait un petit nombre de bandes pour son propre compte . $^{75}$. Il pouvait ensuite les vendre au marché, ou à domicile, pour faire un profit ou encore les échanger contre du petit bétail ${ }^{76}$. De plus, pendant toute la durée de son temps de travail, la cliente fournissait chaque jour un repas au tisserand ${ }^{77}$. Lors de cette transaction, la réputation de la femme pouvait être en jeu. Un ancien tisserand se souvient que «si une femme n'apportait pas à manger au tisserand, tout en travaillant [il] chantait des insultes sur son nom. ${ }^{78}$. Un second explique que les tisserands enlevaient les petits tampons qui se trouvent dans la navette (de part et d'autre de la canette) pour que le rythme et leur chant s'entende de loin ${ }^{79}$.

\section{Trajectoire de la filière cotonnière et textile (du début à la fin du $x x^{e}$ siècle)}

\section{La production textile dendi face aux textiles hausa}

Pour le Dendi, les données ne suffisent pas pour chiffrer la production cotonnière et textile durant la période préindustrielle. Cependant, l'importance sociale du filage, décrite ci-dessus et qui transparait systématiquement dans tous les témoignages de nos interlocutrices, place cette activité au premier rang des préoccupations féminines. Par comparaison avec les centres de production du Sokoto voisin, dont les produits étaient exportés sur plusieurs centaines de kilomètres (e.g. Kriger 1993, Shea 2006), la production textile dendi avait une échelle restreinte, reposant sur un réseau villageois. Dans chaque village, on trouvait quelques tisserands installés en atelier en dehors du village. Les rangs de ces ateliers étaient ponctuellement grossis par la venue de tisserands saisonniers venus du Dallo Bosso (Niger) ou du Borgou (Benin) et pouvaient alors compter jusqu'à une vingtaine d'artisans travaillant en parallèle ${ }^{80}$ (Smolderen 2018).

Cette production modeste a résisté pendant un temps à la concurrence. Dès la fin du $\mathrm{XIX}^{\mathrm{e}}$ et durant la première moitié $\mathrm{du} \mathrm{xx}^{\mathrm{e}}$ siècle, des caravanes hausa importaient plusieurs produits du Nigéria voisin, parmi lesquels des tissus «noirs brillants $»^{81}$ teints à l'indigo ${ }^{82}$. «Pour éviter les douanes des blancs, ces marchands se déplaçaient surtout la nuit. $»^{83}$. Ce témoignage nous conduit à penser qu'il s'agissait plutôt de groupes de colporteurs hausa et non de larges caravanes, lesquelles ne pouvaient se déplacer la nuit sans attirer l'attention des douaniers. Nos données restent lacunaires à leur sujet. Il semble toutefois que ces produits en apparence similaires (pagnes noirs teints à 
l'indigo) n'aient jamais véritablement concurrencés les pagnes produits localement. La production de tissus teints dans le Dendi était bien rôdée. Un réseau d'ateliers de teinture à l'indigo (cf. Figure 2) permettait aux habitants de la région de s'approvisionner facilement en tissus noirs. Afin d'assurer la pérennité de leur activité, les teinturiers ont mis en place un service apparenté à une livraison à domicile ${ }^{84}$. Lorsque leur puits de teinture étaient prêts à l'emploi, ils déambulaient de village en village pour collecter les fils et pagnes à teindre et les ramenaient à leurs ateliers, évitant de cette manière que leur clientèle se fournisse auprès des marchands ambulants. Une fois l'ouvrage terminé, les fils ou pagnes teints étaient rapportés à leurs propriétaires. Parfois un rendez-vous entre le teinturier et le client était fixé au prochain jour de marché ${ }^{85}$, ce dernier y récupérait alors sa commande.

Les pagnes produits localement servaient à l'habillement de tous les jours. Or les textiles importés du Sokoto étaient, quant à eux, probablement l'apanage des chefs et notables, soucieux de pouvoir s'aligner sur un style vestimentaire élitiste et musulman (supra). À l'inverse des pagnes produits localement qui s'échangeaient contre un surplus de fil, ou parfois contre des bottes de mil, le propriétaire d'un pagne importé du Sokoto montrait ostensiblement sa capacité à s'intégrer dans une économie de marché. Les textiles hausa et les pagnes produits localement ne jouaient donc pas dans la même catégorie ${ }^{86}$.

\section{De l'artisanat local à l'économie de marché}

71 Un autre acteur a, quant à lui, durablement affecté l'ensemble de la filière textile. Après la première guerre mondiale, l'administration coloniale poursuit les recherches sur le coton. Des études en tout genre se multiplient87 (Levrat 2008: 55-57). À partir des années 1920-30, les efforts coloniaux visant à exploiter de manière systématique cette ressource dans l'ensemble de l'Afrique Occidentale Française (AOF) résonnent jusque dans le nord du Dahomey (Reste 1934: 131, Levrat 2008: 125-126). J.-F. Reste, gouverneur du Dahomey de 1928 à 1930, remarque qu'une grande partie des terres de la colonie offrent de belles perspectives en matière de coton et est pourtant restée jusquelà inexploitée (Reste $1934: 130$ ).

Cette période voit se mettre en place les premiers essais cotonniers (supra). On cherche à rationaliser la culture cotonnière (trouver une variété adaptée à chaque région en veillant à éviter tout phénomène d'hybridation entre les zones) et à améliorer la production (obtenir à une qualité de fibres répondant aux standards de l'industrie textile européenne). Dans les années 1930, après plusieurs essais, c'est finalement le Gossypium hirsutum (var. Allen) qui est sélectionné et introduit dans la région. Cette dernière espèce avait obtenu de bons résultats au Nigéria à la même latitude et semblait donc capable de s'adapter au climat de cette zone septentrionale (Reste 1934 : 131, 136).

Pour assurer la réussite de son projet cotonnier, l'administration coloniale devait s'assurer que le coton était bien vendu aux négociants français et non écoulé dans le circuit local. Relativement peu d'informations nous sont parvenues à propos des modalités de cette transition entre la culture artisanale pérenne et la culture commerciale. Un seul ancien tisserand a évoqué les difficultés auxquelles il avait pu faire face : 
«Des militaires qui travaillaient pour les blancs vérifiaient qu'on leur vendait bien tout notre coton, pour éviter qu'on l'utilise pour nos propres besoins. Puis il fallait payer l'impôt, même un tisserand devait payer 1000 frs, alors que ce métier ne rapporte pas assez. Petit à petit, on a laissé nos métiers et on a fait l'agriculture pour pouvoir payer l'impôt et éviter les amendes. ${ }^{88}$. Ces méthodes coercitives démontrent l'intérêt de la France pour cet «or blanc ». Elles participent également à transformer les représentations collectives qui l'entourent. Le coton n'est plus perçu comme une fibre à tisser, petit à petit il devient « une valeur ».

D'autre part, il fallait aussi que les voies de communication entre le Nord et le Sud du pays permettent un acheminement aisé de la production cotonnière vers le littoral, en vue de son exportation vers la métropole (Reste 1934 : 179). Dès 1899 émerge le rêve colonial de construire un réseau de voies ferrées qui permette la communication entre ces territoires coloniaux et l'acheminement des ressources de l'intérieur des terres vers les littoraux. Des quatre réseaux envisagés dans ce projet, la voie reliant Cotonou au Niger est la plus courte (Tixier 1965 : 918). Mais pour des raisons financières, le projet n'aboutira finalement jamais. La voie ferrée s'arrête définitivement à Parakou, qui devient alors un point de rupture de charge. Plus au Nord, il faut se contenter du réseau routier pour le transport des ressources (Brégand 1998 : 196).

De grands travaux d'aménagements sont initiés à partir des années 1920 (Van Driel 2001 : 56). Avec l'amélioration des routes, la circulation des marchandises devient plus aisée. Petit à petit, on voit apparaître sur les marchés dendi des produits d'importations. Dans les années 1940, les «fella" (bassines en cuivre d'abord, puis émaillées) font partie des premiers objets de facture industrielle que l'on trouve dans le Dendi. Ces objets venus du Nigéria sont rapidement devenus les pièces maitresses des trousseaux de mariage ${ }^{89}$.

77 Dans le courant des années 1950, les pagnes industriels font progressivement leur apparition sur les marchés. Des colporteurs dendi se spécialisent dans leur transport ${ }^{90}$. Ils descendent jusqu'à Parakou ou se rendent à Niamey pour ramener ces pagnes aux couleurs chatoyantes et aux prix démocratiques. D'après un témoignage, dans les années 1965 , les pagnes industriels s'achetaient $125 \mathrm{~F}$ sur le marché alors que les pagnes artisanaux étaient vendus $300 \mathrm{~F}^{91}$. Cette fois, la concurrence pour les tisserands est rude : le public visé par les textiles industriels est le même que celui des tisserands locaux.

78 Principales clientes, les femmes apprécient tout particulièrement les productions industrielles, moins lourdes et plus faciles à laver que les pagnes tissés à la main ${ }^{92}$. Cependant, l'arrivée des textiles de facture industrielle n'a pas marqué l'arrêt définitif de la production locale. Celle-ci s'est maintenue pendant plus de trente ans après leur arrivée sur les marchés locaux. Dans d'autres régions d'Afrique de l'Ouest, une situation similaire avait déjà été observée. Au Soudan français (actuel Mali) par exemple, l'importation d'étoffes a commencé dès la fin du xix ${ }^{e}$ siècle. Cependant, les efforts français pour développer la culture et l'exportation cotonnière n'ont pas abouti. Au contraire, comme le souligne Roberts, durant toute la période d'entre-deux guerres l'artisanat textile local a non seulement tenu tête aux importations, mais plus encore, la production locale a augmenté (Roberts 1996 : 192-220).

Deux autres objets apparaissent également sur les marchés et vont eux aussi affecter la filière textile : les boloti (les pelotes de fil industrielles) et les cardes manuelles ${ }^{93}$. Les pelotes de fil viennent du Nigéria ou du Niger et offrent aux tisserands une plus grande 
palette de couleurs. Elles leur permettent d'expérimenter mais aussi de répondre à la demande grandissante des clientes pour des couvertures et des pagnes colorés, particulièrement appréciés pour la constitution des trousseaux de mariage ${ }^{94}$. Les cardes manuelles, quant à elles, arrivent de Niamey, du Burkina, de Malanville, et remplacent très rapidement l'arc à battre le coton ${ }^{95}$ (cf. Figure 3). Elles permettent en un temps réduit de démêler et d'aligner les fibres de coton. L'usage de ces cardes manuelles représente un gain de temps pour les fileuses. L'opération de cardage est plus rapide que celle du battage et les fibres obtenues sont alignées et parallélisées, ce qui les rend plus faciles à filer. Ces deux objets - les boloti et les cardes manuelles - apportent donc une aide notable à la filière textile qui se voyait concurrencer par l'arrivée des pagnes industriels (Roberts 1996 : 96).

Figure 5 : Un ancien tisserand porte un pagne de mariage doussa, réalisé grâce aux fils industriels (boloti)
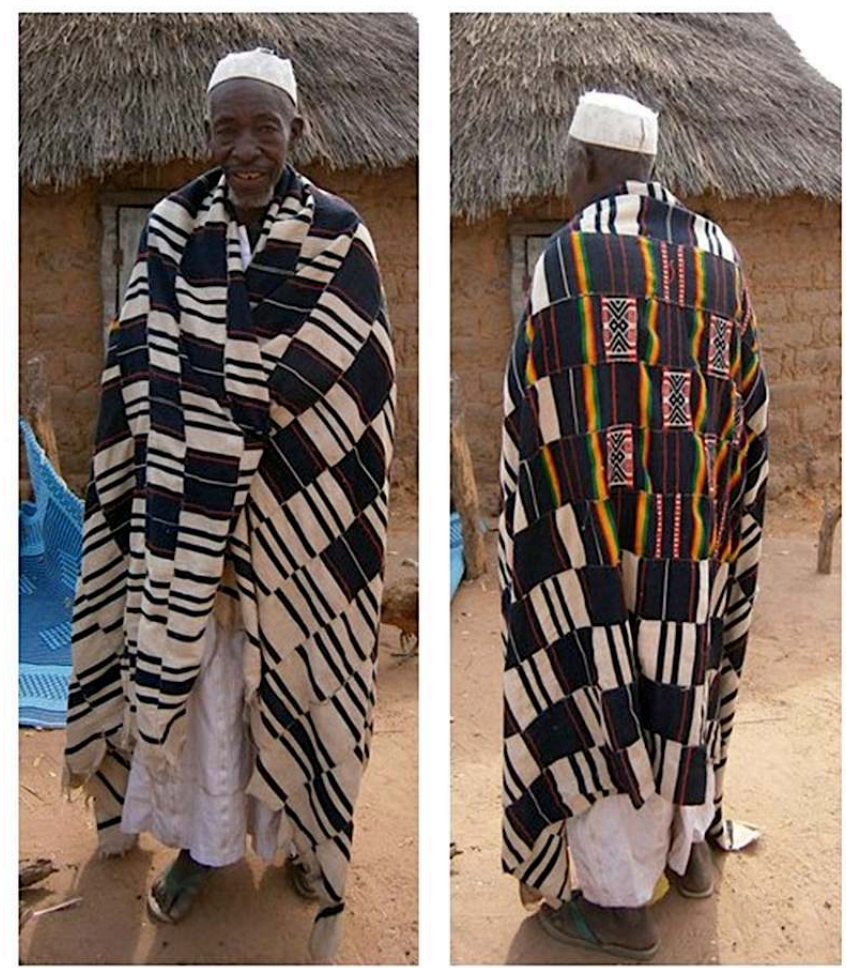

Il le porte à la manière d'un jeune marié, les motifs géométriques dans le dos. Par la suite ce pagne servait à décorer la chambre de la jeune mariée mais pouvait aussi être porté lors des grandes occasions

(c) L. Smolderen (2013)

Dans les années 1950-60, la filière connait deux tendances divergentes. L'arrivée des tissus de facture industrielle sur les marchés met à mal la production locale. Mais parallèlement, l'arrivée des fils de couleurs et des cardes manuelles lui donne un nouveau souffle. Les boloti surtout, permettent aux tisserands dendi de réaliser à moindre frais des pagnes de mariage aux motifs géométriques complexes (Figure 3). Ceux-ci s'inspirent des textiles confectionnés par les tisserands zarma et peul du Niger ${ }^{96}$ (Anquetil $1977:$ 37-40, Picton \& Mack 1979, Etienne-Nugue \& Saley $1987: 191$, 198). Les tisserands dendi ont appris à réaliser les décors géométriques auprès des exodants nigériens, venus travailler sur la rive droite du fleuve pendant la saison 
sèche ${ }^{97}$. De nombreuses jeunes femmes zarma, hausa et peul originaires du Niger se sont mariées dans le Dendi béninois, emmenant avec elles leurs trousseaux de mariage, au sein duquel les pagnes aux décors géométriques occupent une place centrale ${ }^{98}$. Ces pagnes étaient portés par les époux le jour du mariage, ils servaient par la suite à décorer la chambre de la mariée ${ }^{99}$. La circulation des artisans et les liens matrimoniaux entre les villages béninois de la vallée du fleuve et les villages du sud du Niger (région du Dallol Bosso, du Boboye et du Dallol Fogah notamment) ont favorisé l'alignement des critères d'appréciation stylistiques des femmes du Dendi sur ceux de leurs voisines nigériennes.

81 Influencées par les trousseaux de mariage peul, zarma et hausa (Gosselain et al. 2009), le statut des trousseaux dendi s'est transformé100. D'un assemblage d'objets utilitaires, durant la première moitié $\mathrm{du} \mathrm{xx}^{\mathrm{e}}$ siècle, les objets sont de plus en plus orientés vers la décoration de la chambre (fig. 4). Derrière la décoration de la chambre, l'enjeu social est grand $^{101}$. Les chambres des jeunes mariées font l'objet de l'attention de tous et, surtout, de toutes. Le moindre détail est observé. Les assemblages de trousseaux répondent à des critères spécifiques qui permettent de juger du statut de la jeune mariée, et par extension celui de sa famille et de tout son réseau (Gosselain et al. 2009, Cooper 1997) ${ }^{102}$. De nos jours, les pièces maîtresses des trousseaux de mariage sont les petites casseroles émaillées, empilées les unes sur les autres (cf. Figure 6). Plusieurs critères entrent en jeu dans l'évaluation de ces pièces, comme leur couleur et leur poids. Mais surtout c'est l'accumulation de pièces identiques, ou du moins semblables, qui est valorisée au sein des trousseaux. D'autres éléments viennent également augmenter la valeur d'un trousseau, comme le mobilier (des étagères pour disposer les casseroles, des chaises, des fauteuils, un épais matelas en mousse), le textile (des tentures sur les murs, des couvertures épaisses en velours et aux motifs orientaux), mais aussi des glacières ou des casseroles en inox qui ne monteront jamais sur le feu. Autant d'éléments qui symbolisent la modernité et l'opulence. 
Figure 6 : Le trousseau contemporain d'une jeune mariée

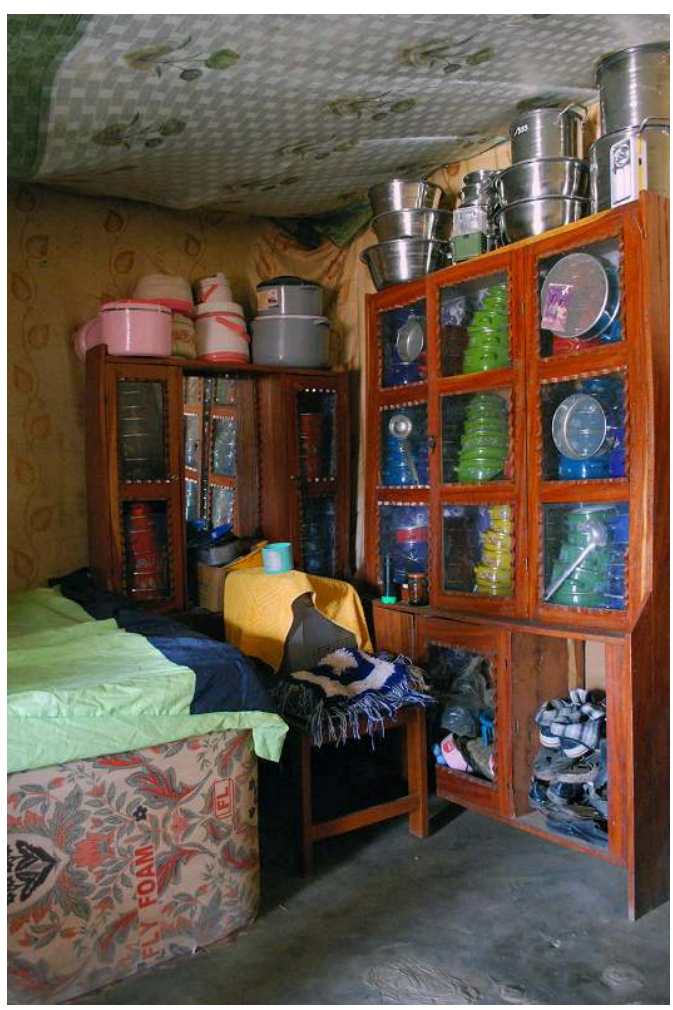

Ce dernier répond aux critères actuels d'accumulation et d'empilement de tasses (casseroles). On remarque également le mobilier en bois, le matelas en mousse, les glacières en plastique et les pièces en inox au-dessus de l'étagère, autant de symboles de modernité

(c) L. Smolderen (2015)

Progressivement, les femmes se mettent à "investir " dans les trousseaux de leurs filles. L'argent de la compensation de mariage ne suffit plus pour couvrir le frais ${ }^{103}$. Dans les années 1950-60, cette tendance se fait déjà sentir et le textile offre alors de nouvelles possibilités : celle de s'enrichir. En plus du fil destiné à l'habillement de la famille, les femmes filent un surplus de bobines afin de fabriquer des pagnes destinés à la vente. Elles se rendent elles-mêmes sur les marchés ou envoient leurs maris réaliser les transactions. Le profit sert en cas de coups durs au sein du ménage mais, surtout, il est investi dans les trousseaux de leurs filles ${ }^{104}$. Le changement de statut des trousseaux illustre la trajectoire socio-économique des femmes qui, dès les années 1950-60, s'intègrent petit à petit dans une économie de marché. Cependant, l'engouement généralisé pour ces pagnes chatoyants aux décors géométriques n'a pas été suffisant pour maintenir la production textile artisanale face à la concurrence des pagnes industriels. Progressivement, le décor des chambres se tourne vers l'accumulation et les empilements de bassines et casseroles émaillées. Les pagnes tissés disparaissent des chambres des jeunes mariées.

Parallèlement à l'arrivée des produits d'importations sur les marchés, les efforts de l'état colonial d'abord, puis ceux de l'état indépendant après 1960, pour sécuriser le secteur cotonnier ne faiblissent pas. La création de l'Institut de recherches du coton et des textiles exotiques (IRCT) en 1946, suivie de près par celle de la Compagnie française pour le développement des fibres textiles (CFDT) en 1949 marque l'installation d'un programme cotonnier à l'échelle de l'AOF (e.g. Levrat 2009, Kpadé \& Boinon 2011). Dans la vallée béninoise du Niger, il faut attendre les années 1960 et l'introduction de la 
charrue tractée par les bœufs pour que s'intensifie la culture cotonnière (Van Driel 2001 : 106-107). Ce n'est finalement qu'à partir des années 1970 que les rendements augmentent véritablement dans la vallée béninoise du fleuve, grâce notamment aux soutiens financiers apportés par l'état et à la mise en place des Centres d'actions régional pour le développement rural (CARDER) (Van Driel 2001: 112-113). Progressivement, le coton change de statut: d'une fibre destinée à la production artisanale de pagne, il devient l'or blanc du marché international. Les fileuses, les tisserands et les teinturiers se tournent vers des activités génératrices de revenus comme le commerce, la culture de rente, le maraîchage (e.g. Bastin 2009). Les différents pans de la filière textile dendi s'effritent.

\section{Épilogue}

84

"Aujourd'hui, nos filles ne veulent pas des objets des vieilles dans leur trousseaux. ${ }^{105}$; «tout le trousseau, c'est des roba (objets en pastiques) et des tasses (casseroles), tout demande de l'argent ${ }^{106}$; «on ne met plus de pagnes des tisserands dans les trousseaux, on utilise les pagnes des blancs. $»^{107}$ (Figure 7). Ces constats sonnent comme le point final de la filière textile. Mais en filigrane, le désintérêt des jeunes générations pour le textile artisanal - tout comme l'émotion perceptible de nos interlocutrices face au monde qui change - montre également à quel point l'objet « coton » (graine, fibre ou textile) était socialisé. Son ancrage dans un univers social était tel qu'il n'a pas pu résister aux transformations socio-économiques de ces dernières décennies. La monétarisation de la société et l'ouverture à l'économie de marché ont profondément changé les aspirations des jeunes mais aussi la place de la femme dans la société.

Pendant la majeure partie du $\mathrm{xx}^{\mathrm{e}}$ siècle, on peut dire que la filière textile était une affaire de femmes ${ }^{108}$ : de la gestion du stock de fibres et de la réserve de graines, au filage, à la commande du pagne et à l'influence sur le style. L'ensemble des étapes stratégiques de la filière reposaient sur leur expertise. Le filage était au cœur même de la construction de l'identité féminine, tandis que les pagnes occupaient une place prépondérante dans les trousseaux de mariage. Le port des vêtements était une manière de se situer socialement et, de ce fait, donnait à la femme un rôle crucial dans l'ancrage social du ménage.

Dans les années 1950-60, on l'a vu, plusieurs facteurs extérieurs affectent la filière et en diminue la productivité. Mais les femmes maintiennent leur intérêt pour le textile qui est toujours intégré dans les trousseaux de mariage et la production se maintient à petite échelle. Finalement, c'est dans les années 1980 que la production textile locale prend réellement fin. À cette époque, les femmes se tournent vers l'agriculture. Elles accèdent à plus de liquidité et, avec l'ouverture à une économie de marché néolibérale, leurs représentations sociales changent elles aussi. Les critères de la femme «bonne à marier " se transforment et s'établissent dorénavant dans le champ, tandis que les trousseaux de mariage évoluent en adéquation avec ces nouvelles représentations ${ }^{109}$. Les motifs géométriques tellement appréciés quelques décennies auparavant tombent en désuétude et les critères d'appréciation des décorations de chambre se tournent vers l'accumulation de casseroles empilées, devenue le symbole de la réussite économique. Ancré dans un maillage serré de représentations sociales héritées d'un temps révolu, le textile artisanal n'a plus lieu d'être.

Revue d'ethnoécologie, 15 | 2019 
Figure 7 : À l'arrière de la moto, une jeune mariée porte un pagne de mariage de facture industrielle

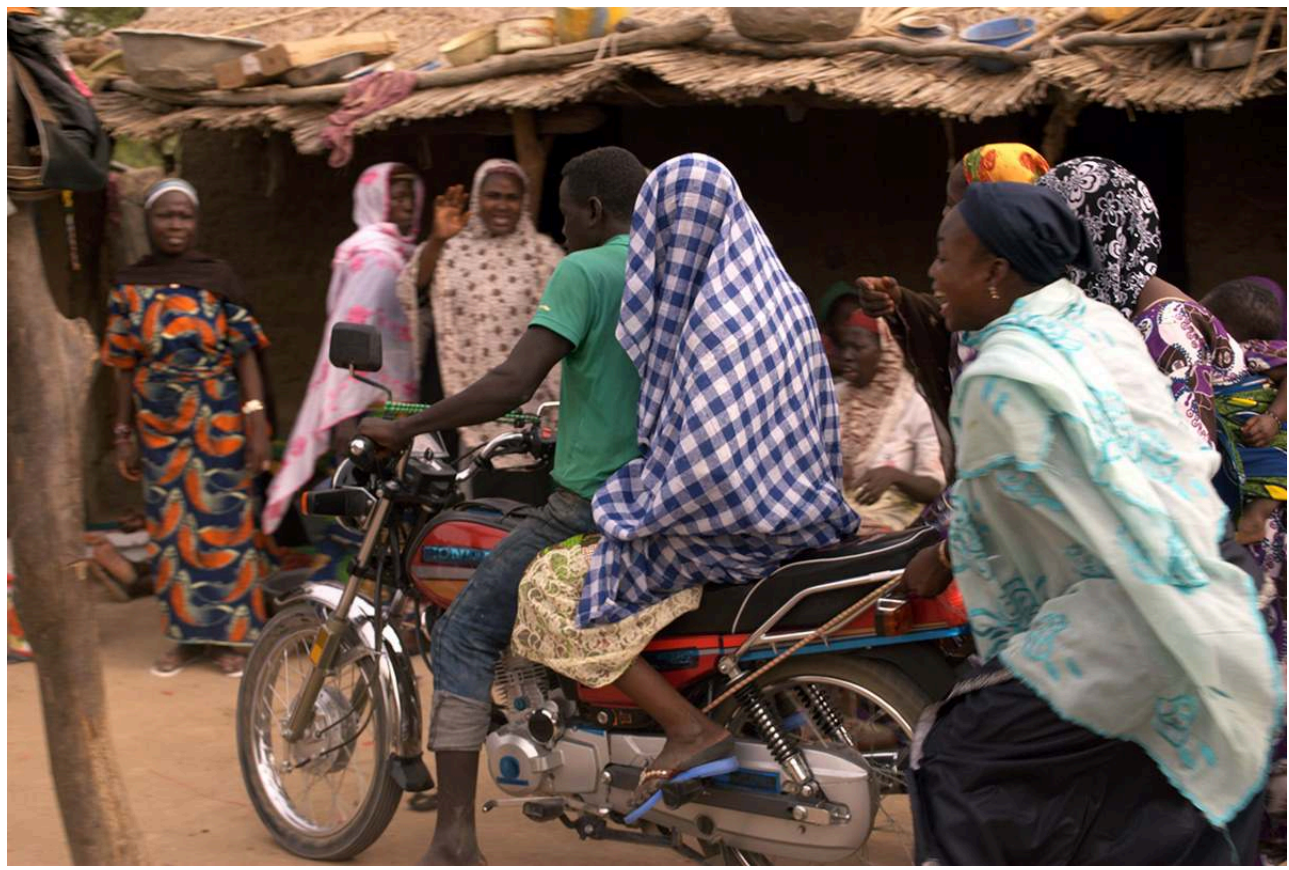

On retrouve le décor en damier qui rappelle celui du pagne artisanal (voir fig.3). La jeune mariée est amenée chez son mari. Elle portera ce pagne pendant une semaine, puis il servira de couvre-lit (c) L. Smolderen (2015)

\section{BIBLIOGRAPHIE}

Adjakpa T.T. 2016 - Gestion des risques hydro-pluviométriques dans la vallée du Niger au Bénin. Thèse, Université d'Abomey-Calavi.

Adjovo-Ahoyo N., Glin L. C. \& Azandegbe D. 2013 - Analyse des interventions du pouvoir public dans le secteur agricole au Bénin de 1960 à nos jours. Étude réalisée pour le Conseil d'analyse économique (République du Bénin).

Ale G.A. 2008 - Libéralisation agricole et incidence sur la production du coton au Bénin. Alternatives Sud $15: 89-105$.

Anquetil J. 1977 - Niger. L'artisanat créateur. Paris, Dessain et Tolra.

Barth H. 1860 - Voyages et découvertes dans l'Afrique septentrionale et centrale. Tome 1. Paris, A. Bonhé.

Bastin S. 2009 - Diffusion du maraîchage féminin et grands espaces de mobilité dans la vallée du Niger. Techniques et Culture $51: 124-143$

Bonnot T. 2018 - Anthropologie et culture matérielle : que faire des choses ? In : Bourgeois L., Alexandre-Bidon D., Feller L., Mane P., Verna C. \& Wilmart M. (Ed.), La culture matérielle : un objet en question. Anthropologie, archéologie et histoire. Caen, Presses Universitaires de Caen : 175-185 
Boussari Vokouma J. 1999 - Les techniques du tissage au Moogo : origines et évolutions. Unpublished $\mathrm{PhD}$ thesis, Université de Provence, Aix-Marseille.

Brégand D. 1998 - Commerce caravanier et relations sociales au Bénin. Les Wangara du Borgou. Paris, L'Harmattan.

Champion L. 2019 - The Evolution of Agriculture, Food and Drink in the Ancient Niger River Basin. Archaeobotanical Studies from Mali and Benin. Unpublished PhD thesis, UCL, London.

Champion L. \& Fuller D. 2018 - Archeobotanical Remains. In : Haour A. (Ed.), Two Thousand Years in Dendi, Northern Benin. Archaeology, History and Memory. Leiden, Brill : 216-233.

Cooper B.M. 1993 - Cloth, Commodity Production, and Social Capital: Women in Maradi, Niger 1890-1989. African Economic History 21 : 51-71.

Cooper B.M. 1997 - Marriage in Maradi. Gender and Culture in a Hausa Society in Niger, 1900-1989. Portsmouth, Heinemann.

De Haan L.J. (sous la dir.) 1997 - Agriculteurs et éleveurs au Nord-Bénin. Écologie et genres de vie. Paris, Karthala.

Duval C. 1864 - La question cotonnière. Paris, Cosson et Co.

Étienne M. 1977 - Women and Men, Cloth and Colonization: The Transformation of ProductionDistribution Relations among the Baule (Ivory Coast). Cahiers d'études africaines 65 (17) : 41-64.

Étienne-Nugue J. 1984 - Bénin. Dakar, Institut culturel africain.

Étienne-Nugue J. \& Saley M. 1987 - Artisanats traditionnels. Niger. Dakar, Institut Culturel Africain.

Forbes R.-H. 1926 - Le coton dans la vallée moyenne du Niger. Essai de culture (1923-1924). Paris, Librairie Émile Larose.

François G. 1906 - Notre colonie du Dahomey, sa formation - son développement - son avenir. Paris, Librairie Emile Larose.

Gbinsa K.Y. 1991 - De fils et de mains: une approche historique du tissage en pays baatonu (bariba). Unpublished Master thesis, Université Nationale du Bénin, Abomey-Calavi.

Gosselain O.P. \& Smolderen L. 2016 - Les fantômes du Dendi. Lorsque surgissent les restes d'une ancienne filière textile au Nord Bénin. Techniques et Culture 65-66 : 452-469.

Gosselain O.P. \& Smolderen L. 2018 - Crossing Archaeology and Oral Tradition: Approaching Dendi History from Sites of Memory. In : Haour A. (Ed.), Two Thousand Years in Dendi, Northern Benin. Archaeology, History and Memory. Leiden, Brill : 6-19.

Gosselain O.P., Zeebroek R. \& Decroly J.-M. 2009 - Les tribulations d'une casserole chinoise au Niger. Techniques et Culture 51 (1) : 18-49.

Haour A. (Ed.) 2018 - Two Thousand Years in Dendi, Northern Benin. Archaeology, History and Memory. Leiden, Brill.

Henry Y. 1906 - Le coton dans l'Afrique Occidentale Française. Paris, Augustin Challamel.

Kpadé P.C. \& Boinon J.-P. 2011 - Dynamique des politiques cotonnières au Bénin. Une lecture par la dépendance de sentier. Économie rurale 321: 58-72.

Kriger C.E. 1993 - Textile Production and Gender in the Sokoto Caliphate. The Journal of African History 34 (3) : 361-401.

Kriger C.E. 2006 - Cloth in West African History. Oxford, Altamira Press. 
Lamb V. 1975 - West African Weaving. Londres, Duckworth.

Lanoye F.T. (de) 1860 - Le Niger et les explorations de l'Afrique centrale. Depuis Mungo Park jusqu'au Docteur Barth. Paris, Hachette.

Lave J. \& Wenger E. 1991 - Situated Learning. Legitimate peripheral participation. Cambridge, Cambridge University Press.

Lemonnier P. 2010 - L'étude des systèmes techniques. Une urgence en technologie culturelle. Techniques et Culture 54-55 : 46-67.

Levrat R. 2008 - Le coton en Afrique occidentale et centrale avant 1950 : un exemple de la politique coloniale de la France. Paris, L'Harmattan.

Levrat R. 2009 - Le coton dans la zone franc depuis 1950. Un succès remis en cause. Paris, L'Harmattan.

Magnavita S. 2018 - Beads and Pendants. In : Haour A. (Ed.), Two Thousand Years in Dendi, Northern Benin. Archaeology, History and Memory. Leiden, Brill : 199-204

Martinière H.-P. de la (sous la dir.) 1898 - La mission Bretonnet. Bulletin du comité de l'Afrique française 3 : 91-96.

Monteil C. 1927 - Le coton chez les Noirs. Paris, Librairie Émile Larose.

Naji M. 2009 - La formation de la féminité à travers le tissage dans le Sirwa (Maroc). In : Julien M.P. \& Rosselin C. (Ed.) Le sujet contre les objets, tout contre : ethnographies de cultures matérielles. Paris, Comité des travaux historiques et scientifiques : 243-263.

Olivier de Sardan J.-P. 1976 - Quand nos pères étaient captifs... : récits paysans du Niger. Paris, Nubia.

Olivier de Sardan J.-P. 1984 - Les sociétés songhay-zarma : Niger-Mali. Paris, Karthala.

Picton J. \& Mack J. 1979 - African Textiles: Looms, Weaving and Design. Londres, British Museum Publications for the Trustee of the British Museum.

Reste J.-F. 1934 - Le Dahomey. Réalisations et perspectives d'avenir. Paris, Comité de l'Afrique Française.

Réteaud L. 1924 - Le progrès de la culture du Cotonnier au Dahomey. Revue de botanique appliquée et d'agriculture coloniale $31(4): 209-212$.

Robert L. 1931 - La culture du coton en Afrique Occidentale Française. Paris, Domat-Montchrestien.

Roberts R.L. 1996 - Two Worlds of Cotton. Colonialism and the Regional Economy in the French Soudan, 1800-1946. Sandford, Standford University Press.

Sainlaude S. 2011 - La France et la confédération sudiste (1861-1865). Paris, L'Harmattan.

Schaedler K.-F. 1987 - Le tissage en Afrique au Sud du Sahara. Munich, Panterra.

Shea P.J. 2006 - Big is Sometimes Best: The Sokoto Caliphate and Economic Advantages of Size in the Textile Industry. African Economic History 34 : 5-21.

Smolderen L. 2018 - Textile Production in Dendi: An Ethnographic and Historical Study of a Chain of Production. In : Haour A. (Ed.), Two Thousand Years in Dendi, Northern Benin. Archaeology, History and Memory. Leiden, Brill : 73-82.

Smolderen L. \& Minguet R. 2013 - Un fil d'Ariane dans le Dendi. Ethnographie d'une technique disparue. Techniques \& Culture 61 (2) : 304-317.

Tchibozo R. 2018 - Figurines and Terracotta Objects. In : Haour A. (Ed.), Two Thousand Years in Dendi, Northern Benin. Archaeology, History and Memory. Leiden, Brill : 210-215. 
Tixier G. 1965 - La succession de la Régie des chemins de fer de l'A.O.F. Problèmes posés par l'apparition de nouveaux États. Annuaire Français de Droit International 11 : 916-932.

Ton P. 2004 - La production du coton au Bénin. Étude réalisée pour le programme « Renforcement des capacités commerciales » de la Fédération Internationale des Producteurs Agricoles.

Tossou R.C. 1995 - People's response to policy change in agricultural development organization. The Benin case. Thèse de doctorat, Wageningen Agricultural University.

Toutée G.J. 1897 - Principaux résultats géographiques de la mission Toutée. Annales de Géographie $6(26): 133-146$.

Toutée G.J. 1899 - Du Dahomé au Sahara. L'homme et la nature. Paris, Armand Colon.

Trouillet J.-P. (sous la dir.) 1905 - De l'océan au Niger par le Dahomey. Dépêche Coloniale Illustrée 5 (9) : 117-128.

Vallard A. 2012 - De la jupe à la femme. Tissage, vêtement et subjectivation au Laos. In : Anstett Gessat E. \& Gelard M-L. (Ed.), Les objets ont-ils un genre ? Paris, Armand Colin : 105-120.

Van Driel A. 2001 - Sharing a Valley. The changing relations between agriculturalists and pastoralists in the Niger Valley of Benin. Leiden, African Studies Centre.

Wennink B., Meenink J.W. \& Djihoun M. (Ed.) 2013 - La filière coton tisse sa toile au Bénin. Les organisations de producteurs étoffent leurs services aux exploitations agricoles familiales. Cotonou/ Amsterdam, KIT Publishers.

\section{NOTES}

1. Pour plus d'informations au sujet de l'hydrographie et de la pédologie de la région voir Adjakpa 2016.

2. Programme de Renforcement des Organisations de Producteurs de Coton

3. Ce projet a bénéficié d'un financement du European Research Council, Septième programmecadre (FP/2007-2013) / ERC Grant Agreement 263747. Ce projet a pour vocation d'approcher l'histoire longue du Dendi, jusqu'alors peu connue, en s'appuyant sur une approche multidisciplinaire archéologique, géologique, historique et ethnographique (Haour 2018).

4. Ce volet d'enquête est un travail en cours mené par Olivier Gosselain (ULB). Voir notamment Gosselain \& Smolderen 2018.

5. La filière compte quatre étapes techniques: la culture du coton, le filage, le tissage et la teinture. L'étape du tissage a été détaillée ailleurs (Smolderen 2018) et celle de la teinture fait l'objet d'un travail de recherche en cours mené par Olivier Gosselain. Ces deux étapes ne seront donc pas détaillées ici.

6. Dans la suite du texte, les références aux enquêtes orales suivent le modèle suivant : date, nom du village, genre de l'informateur (h ou f), âge (x si inconnu).

7. De 2012 à 2015, ces recherches ont été menées dans le cadre du projet Crossroad of Empires dirigé par Anne Haour (UEA). De 2015 à 2018, elles ont été conduites dans le cadre de ma thèse de doctorat.

8. Les travaux de Champion L. ont fait l'objet d'une thèse de doctorat déposée à UCL (Londres) en 2019, Champion 2019.

9. Le second volet de nos recherches de terrain portait sur «le paysage gastronomique» du Dendi.

10. La Sénégambie recouvre une réalité territoriale aux limites floues. Cette zone correspond globalement aux bassins des fleuves Sénégal et Gambie. 
11. Le Moyen-Dahomey correspond à une large zone comprise entre les $7^{\circ}$ latitude nord et $10^{\circ}$ latitude nord (au sud de Kandi).

12. Zone située au Nord du $10^{\circ}$ latitude nord.

13. Le terme dendi habou est le terme générique signifiant « coton ». Sando est un adjectif que l'on peut traduire par « dur».

14. 15.11.15, Kargui, h, 90 ; 17.11.15, Karimama, h, $89 ; 18.11 .15$, Kofouno, groupe f, entre 70 et 80 ; 18.08.16, Isséené, f, $90 ; 18.08 .16$, Torozougou, f, $85 ; 29.11 .15$, Kantoro, h, $75 ; 05.03 .17$, Pekinga, h, 70 ; 17.03.17, Kompanti, h, 70 ; 20.03.17, Kargui, f, x ; 25.02.18, Sia, f, 57 ; 02.03.18, Birni Lafia, f, 75.

15. 14.11.15, Kantoro, h, $80 ; 29.11 .15$, Kantoro, h, 75.

16. 14.11.15, Kantoro, h, $80 ; 14.11 .15$, Torozougou, h, $75 ; 18.11 .15$. Kofouno, groupe f, entre 70 et 80 ; 08.12.15, Garou, h, 75 ; 30.03.16, Koki, h, 65 ; 20.06.16, Sendé 1, f, $65 ; 13.07 .16$, Garou, h, 70 ; 17.03.17, Kompanti, h, 70 ; 26.03.17, Houna, h, 65 ; 31.03.17, Garou, h, 75.

17. 08.12.15, Garou, h, 75.

18. 05.03.17, Pékinga, h, 75.

19. 14.11.15, Kantoro, h, 80.

20. Ce mode de culture pérenne en champ « spécial », et non intercalaire, utilisé dans le Dendi et décrit par Barth au Niger, avait également été observé par le Docteur Yves Henri dans le Yatenga (Haute-Volta, actuel Burkina Faso). Il remarque à ce sujet : « Nulle part le cotonnier n'est cultivé comme plante annuelle; le noir n'arrache jamais les plants, ceux-ci végètent pendant quatre ou cinq ans et sont remplacés au fur et à mesure de leur disparition. On rencontre ainsi dans un même champ, des cotonniers d'un à quatre ou cinq ans. " (Henry 1906 : 119).

21. 31.03.17, Garou, h, $75 ; 02.04 .17$, Toumboutou, h, 80.

22. 210.02.12, Kargui, h, x ; 7.01.12, Bonwalou, h, 60 ; 26.01.13, Karimama, h, 72 ; 13.03.17, Kotatchi h, 68 et $\mathrm{f}, 78$.

23. 10.02.12, Kargui, h, $\mathrm{x}$; 05.03.17, Pekinga, h, 70 ; 20.03.17, Kargui, f, x ; 26.03.17, Houna, h, 65 .

24. 11.02.12, Molla, h, $70 ; 09.03 .17$, Malanville, $h, x$.

25. 08.12.15, Garou, h, 75.

26. 13.07.16, Garou, h, 70.

27. Champion 2019. Voir aussi les critères descriptifs fournis par PlantUse, disponibles sur: https://uses.plantnet-project.org/en/Gossypium_arboreum_(PROTA), consulté le 30.05.19.

28. 29.11.15, Kantoro, h, 75.

29. 28.11.15, Torozougou, $f, 70$.

30. 13.07.16, Garou, h, 70.

31. 29.01.12, Goumbitchigoura, h, 70 ; 29.01.12 Monsey Dendi, h, 57 ; 31.01.12, Kompa, h, 90.

32. 20.03.17 Kargui, $\mathrm{f}, \mathrm{x}$.

33. 31.03.17, Garou, h, 75.

34. 01.02.13, Birni Lafia, f, 85 ; 22.01.13, Mamassi gourma, h, 80 ; 26.01.13, Karimama, h, 75.

35. 01.02.13, Birni Lafia, f, $85 ; 26.01 .13$, Karimama, h, $72 ; 29.01 .13$, Bonwalou, h, 80.

36. 08.12.15, Garou, h, 75.

37. 30.03.16, Koki, h, 65.

38. 22.01.13, Mamassi Gourma, h, 80 ; 23.01.13, Kofounou, h, x; 26.01.13, Karimama, h, 72 ; 27.01.13, Pekinga, h, 68 ; 17.11.15, Karimama, h, 89 ; 08.07.16, Karimama, h, 95.

39. 22.01.13, Mamassi Gourma, h, 80.

40. 28.01.13, Pekinga, h, $55 ; 13.06 .16$. Karimama, f, 65

41. 22.01.13, Mamassi Gourma, h, 80.

42. Les groupes peuls se trouvent essentiellement dans les villages de Pekinga, Kompanti et Mamassi Peul.

43. 27.01.13, Pekinga, h, 70 ; 28.01.13, Pekinga, h, 75 ; 30.01.13, Kompa, f, 53.

44. 02.02.12, Karimama, groupe h, 49-85; 12.02.12, Kwara Tegui, f, 80 et f, 55 . 
45. 27.01.13, Pekinga, h, 65. Mention est faite de ces "caravanes » dans La Dépêche Coloniale Illustrée (1905 5(9), 125).

46. 07.03.17, Koullou, f, $68 ; 13.03 .17$, Kotatchi, f, $78 ; 27.02 .18$. Tara f, $70 ; 01.03 .18$, Toura, $f, 90$ et f, $80 ; 02.03 .18$, Birni Lafia, f, 70.

47. Une concession est une unité d'habitation. Elle comprend les membres d'une famille élargie. Plusieurs habitations correspondant à des unités familiales nucléaires ou polygames sont regroupées autour d'une cour commune et partagée, généralement clôturée. Chaque père de famille est considéré comme "chef de famille » au sein de son unité familiale, mais le plus âgé des hommes de la concession est considéré comme « le chef de la concession ».

48. 09.09.16, Madékali, f, $80 ; 17.09 .16$, Gouroubéri, f, 80 et f, 90.

49. 27.02.18, Tara, f, 70 .

50. 08.03.17, Albarkaizé, f, 50 .

51. 29.01.13, Bonwalou, f, 75.

52. 29.01.13, Bonwalou, f, $75 ; 02.02 .13$, Karimama, groupe f, 68-73.

53. 03.02.12, Karimama, f, 90.

54. 01.03.18, Toura, $\mathrm{f}, 90$ et $\mathrm{f}, 80$.

55. 10.06.16. Kompanti, f, 85-90

56. 27.02.18. Tara, $\mathrm{f}, 70$.

57. Voir à ce propos les travaux de Naji (2009) et Vallard (2012) portant sur d'autres activités textiles.

58. 12.03.15, Toumboutou, $f, 60$

59. 17.09.16, Gouroubéri, $\mathrm{f}, \mathrm{x}$.

60. 02.02.13, Karimama, groupe f, 68-73;24.09.16, Toumboutou, f, $85 ; 05.03 .17$. Pekinga, f, 60.

61. Le Dendi est resté majoritairement animiste, jusqu'à une époque récente. La conversion à l'Islam remonte aux années 1970 tout au plus. Cependant, les chefs des villages - et particulièrement ceux qui se revendiquent d'une ascendance songhay - ont depuis longtemps associés aux pratiques cultuelles animistes les pratiques maraboutiques islamiques afin d'assurer la pérennité de leur pouvoir. Parallèlement, en ce qui concerne les chefferies songhay, le port d'un vêtement ostensiblement musulman pourrait être une manière de rappeler leur lien historique (du moins supposé) avec la lignée des Askia (noms donné aux empereurs de la dynaste Songhay). Pour plus de détails sur l'histoire de la région se référer aux travaux en cours d'olivier Gosselain.

62. Les textiles teints à l'indigo du Califat de Sokoto avait une couleur noire et brillante. 27.01.13, Pekinga, h, $65 ; 29.01 .13$, Bonwalou, h, $66 ; 30.01 .13$, Kompa, h, 90 et f, 53.

63. 07.03.17, Koullou, f, 68.

64. 25.02.18. Sia, $\mathrm{f}, 57$.

65. Le critère principal de l'évaluation de la qualité d'un textile reposait sur la densité du tissage, particulièrement du tassage de la trame. «Les tissus de mauvaise qualité étaient si peu tassé que l'on voyait presque à travers. » (22.01.13, Kofounou, h, 70).

66. Il s'agit des pagnes de mariage (fig. 4) offerts par la mère de la mariée à son gendre. Ces pagnes étaient ornés de motifs colorés obtenues par teintures naturelles d'abord et plus tard grâce aux fils industriels (boloti), (03.03.18, Birni Lafia, f, 80). Avant les années 1950, période à laquelle ces pagnes se sont démocratisés, les pagnes de mariage étaient des pagnes réservés aux personnes aisées (13.02.15, Birni Lafia, $f, 90)$.

67. 10.02.12, Bini Lafia, h, $66 ; 04.02 .15$, Toumboutou, f, $80 ; .27 .02 .18$, Tara, f, 70 .

68. La tontine est une forme collective d'épargne, de biens (bassines, tasses, pagnes) ou numéraire (somme d'argent prédéfinie). Cependant, avant l'avènement d'une économie monétarisée, le même système existait pour des activités techniques (la fabrication du beurre de karité, le filage). Cette forme de collectivisation de l'effort était typiquement féminine. 
69. 13.06.16. Karimama, f, 65 ; f, 65, 15.06.16. Kargui ; f, 80. 21.06.16. Madékali ; f, 80, 23.08.16. Madécali ; f, 78, 13.03.17. Kotatchi ; f, 70. 27.02.18. Tara.

70. Sur ce type de métier horizontal, le textile est obtenu par l'entrecroisement perpendiculaire du fil de trame et des fils de chaîne tendus horizontalement grâce à une chaîne mobile. De nombreux ouvrages ont traités des aspects techniques spécifiques du tissage sur ce type de métier (e.g. Lamb 1975 ; Picton \& Mack 1979 ; Schaedler 1987 ; Gbinsa 1991 et Boussari Vokouma 1999).

71. 13.06.16. Karimama, f, $65 ; 23.06 .16$, Boïffo, f, 65.

72. Les enquêtes n'ont pas donné d'indication précise quant au nombre de fils de chaîne nécessaires.

73. 08.02.12, Bogo Bogo, h, $70 ; 10.02 .12$, Birni Lafia, h, 66 ; 22.06.16, Gandé Gabi, f, 75.

74. 29.01.12, Goumbitchigoura, h, 70.

75. 28.01.13, Pekinga, h, 65 ; 31.01.13, Kompanti Debere, h, 70.

76. Ce type de transactions étaient courantes avec les groupes peuls qui ne produisaient pas de coton et qui reposaient donc sur l'échange de pagnes ou de bobines de fil pour se vêtir.

77. 01.03.18, Toura, $\mathrm{f}, 90$ et $\mathrm{f}, 80$.

78. 07.03.17, Koullou, h, 70.

79. 06.02.13, Torozougou, h, 70 .

80. 31.01.13, Kompanti Debere, h, 70 ; 06.02.13, Torozougou, h, 70

81. 29.01.13, Bonwalou, h, 66 .

82. 27.01.13, Pekinga, h, $65 ; 30.01 .13$, Kompa, h, 90 et f, 53.

83. 27.01.13, Pekinga, h, 61 .

84. 01.02.12, Kwara Tegui, h, $60 ; 10.02 .12$, Bini Lafia, h, $66 ; 10.02 .12$, Kargui, h, $\mathrm{x} ; 22.01 .13$, Kofounou, h, 70 ; 31.01.13, Bogo Bogo, h, 80 ; 05.02.13, Skawan, h, 68.

85. Les ateliers de teinture étaient souvent associés au village disposant d'un marché. Dans le Dendi, la teinture était le fait de deux segments de populations spécialisés : les Kumaté et les Gulmanceba (voir à ce sujet les travaux en cours d'Olivier Gosselain).

86. 27.01.13, Pekinga, h, 65.

87. Des missions scientifiques appliquées (par exemple Forbes 1926), mais aussi des thèses de doctorat (comme celle de Robert 1931), ainsi que des études à visée plus historique (voir notamment Monteil 1927, cité par Levrat 2008 : 77).

88. 31.01.13, Kompanti Debere, h, 70.

89. 04.12.15, Karimama f, 90 ; 07.06.16, Pekinga, f, 50 ; 21.06.16. Madékali, f, 80 ; 22.06.16. Monkassa, f, 70 ; 23.06.16. Boïffo, f, 60 ; 23.08.16, Madécali, f, $80 ; 02.10 .16$. Molla, f, x.

90. 03.02.12, Karimama, f, $80 ; 07.03 .17$, Koullou, f, 64 .

91. 07.03.17, Koullou, f, 64.

92. 03.02.12, Karimama, f, 80 ; 14.06.16. Birni Lafia, f, 55 ; f, 15.06.16. Kargui, f, 65

93. 29.01.13, Bonwalou, h, 66 ; 31.01.13, Kompanti Debere, h, $70 ; 13.03 .17$. Kotatchi, f, 78 ; 02.03.18, Birni Lafia, f, $75 ; 02.03 .18$, BirniLafia, f, 75 ;

94. 29.01.13, Bonwalou, h, 66 ; 31.01.13, Kompanti Debere, h, 70 ; 06.02.13, Torozougou, h, 70 ; 15.06.16, Kargui, f, $65 ; 07.03 .17$. Koullou, f, 68

95. 13.03.17. Kotatchi, f, $78 ; 03.03 .18$, Birni Lafia, f, 85.

96. 29.01.13, Bonwalou, h, 66 et f, $75 ; 03.11 .15$, Sakawan, f, $60 ; 11.06 .16$. Toryo, f, $70 ; 14.06 .16$. Birni Lafia, f, 60 ; 15.06.16, Kargui, f, 60 ; 27.03.17. Houna, f, 55 ; 12.04.17. Botol, f, 55

97. 29.01.13, Bonwalou, h, 66 .

98. 27.01.13, Pekinga, h, 61 ; 29.01.15, Fulan Baba Koara, f, 54 ; 12.06.16, Mamassi Peul, f, 57 ; 12.06.16, Mamassi Peul, f, $29 ; 25.02 .18$, Sia, f, 54. Il existe plusieurs sous-catégories appartenant à la famille stylistique des pagnes à décors géométriques en fonction du nombre de bandes décorées et de la disposition des motifs. Par exemple, le pagne babaji compte 13 bandes dont 7 portent des décors géométriques, tandis que le pagne doussa (ou dissaré en fulfulde) a 5 bandes 
portant des décors géométriques (07.06.16. Pékinga, h, 83 ; 08.06.16. Pekinga. F, 64). D’autres styles appartenant à cette famille (sakala, dedendi, soubani) existent mais, faute de témoins matériels, ils n'ont pas pu être définis.

99. Aujourd'hui encore dans le village de Mamassi Peul et de Pekinga, deux villages peuls, certaines jeunes filles se servent de pagnes à décors géométriques pour orner leur chambre. Elles les achètent à un exodant nigérien qui les importe de Niamey. 12.06.16, Mamassi Peul, f, 29 ; 05.03.17, Pekinga, f, 32.

100. Au sujet de la trajectoire socio-économique des trousseaux de mariage, dans une autre région, voir Cooper 1997 : 90-109.

101. 30.01.13, Kompa, f, $70 ; 01.02 .15$. Toumboutou, f, 40 .

102. 01.02.15, Toumboutou, $\mathrm{f}, 40 ; 12.02 .15$, Birni Lafia, $\mathrm{f}, 50$;

103. La compensation de mariage a une valeur prédéfinie. Il s'agit d'une somme d'argent (par le passé il pouvait aussi s'agir d'un cheval) donnée par la famille du futur époux à celle de la future mariée. Cette somme (ou la valeur équivalente) servait à acheter les objets du trousseau.

104. 29.01.13, Bonwalou, f, 75, 29.01.13, Loumbou Loumbou, f, 80 ; 31.01.13, Bogo Bogo, f, x ; 13.06.16. Karimama, f, $90 ; 13.06 .16$. Karimama, f, 65 ; 23.06.16. Boïffo, f, 60 ; 27.03.17, Houna, f, 55 ; 25.02.18. Sia, $f, 54$ et $f, 58 ; 01.03 .18$. Toura, $f, 90$ et $f 80$.

105. 07.06.16, Pékinga, $\mathrm{f}, \mathrm{x}$.

106. 09.07.16, Toumboutou, $f, 50$.

107. 29.01.15, Fulan Baba Koara, f, 54.

108. Au sujet de la place des femmes dans la production textile, pour d'autres régions, voir les travaux d'Étienne (1977), Cooper $(1993,1997)$ et Kriger $(1993,2006)$.

109. Ce volet est développé dans la thèse de doctorat de Smolderen (en cours).

\section{RÉSUMÉS}

En suivant la filière textile artisanale (graines, fibres, fils, pagnes) et ses différents acteurs (cultivateurs, fileuses, tisserands, teinturiers), cet article propose une étude micro-historique de la filière textile artisanale du Dendi (Nord-Bénin) de la fin du $\mathrm{XIX}^{\mathrm{e}}$ siècle au milieu du Xx $\mathrm{x}^{\mathrm{e}}$ siècle. Le cadre chronologique limité de l'étude dénote des autres études qui traitent de l'histoire des Gossypium sp., de l'évolution de leurs génomes, ou encore de la diffusion des techniques textiles, lesquelles se développent sur plusieurs siècles (voir ce volume). Le parti-pris de cet article est toute autre. Autour de l'objet coton se déroule une histoire sociale intime - liée à la construction de l'identité féminine et à la trajectoire socio-historique des acteurs de la filière - qui nous éclaire sur les bouleversements récents qu'a connus la région.

This article follows the production of artisanal textiles (seeds, fibres, threads and cloth) and its various actors, offering a micro-historic study of the textile industry in Dendi (North Benin), from the end of the $19^{\text {th }}$ century to the mid- $20^{\text {th }}$ century. This limited chronological framework differs from other studies devoted to the history of Gossypium sp. which explore their genomic evolution or the diffusion of textile techniques over several centuries. This article follows a different approach, going way beyond the materiality of "cotton" alone: an intimate and social history is woven in and around the object "cotton". Its study informs us on the construction of feminine identity and the socio-historical trajectory of the industry's actors, while shedding light on the major changes which have recently taken place in the region. 
INDEX

Keywords : Ethnography, crafts and techniques, cotton agriculture, spinning, colonisation, Dendi, Benin

Mots-clés : Ethnographie des techniques, culture du coton, filage, colonisation, Dendi, Bénin

\section{AUTEUR}

LUCIE SMOLDEREN

Phd-Student, Centre d'Anthropologie Culturelle, CRéA-Patrimoine (Université Libre de Bruxelles) lusmolde@ulb.ac.be 